\title{
The Aromatic Infrared Bands as seen by ISO-SWS: Probing the PAH model ${ }^{\star}$
}

\author{
L. Verstraete ${ }^{1}$, C. Pech ${ }^{2}$, C. Moutou ${ }^{3}$, K. Sellgren ${ }^{4}$, C. M. Wright ${ }^{5}$, M. Giard ${ }^{2}$, A. Léger ${ }^{1}$, \\ R. Timmermann ${ }^{6}$, and S. Drapatz
}

1 Institut d'Astrophysique Spatiale, Bât. 121, Université de Paris XI, 91405 Orsay Cedex, France

2 CESR, 9 avenue du Colonel Roche, 31028 Toulouse Cedex, France

3 Alonso de Cordoba 3107, Santiago 19, Chile

4 Astron. Dept., Ohio State University, 140 West 18th avenue, Colombus OH 43210, USA

${ }^{5}$ Univ. College, ADFA, UNSW Canberra, Australia

6 Max-Planck Inst. für extraterr. Physik, Postfach 1603, 85740 Garching, Germany

Received 2 October 2000 / Accepted 29 March 2001

\begin{abstract}
We discuss the Aromatic Infrared Band (AIB) profiles observed by ISO-SWS towards a number of bright interstellar regions where dense molecular gas is illuminated by stellar radiation. Our sample spans a broad range of excitation conditions (exciting radiation fields with effective temperature, $T_{\text {eff }}$, ranging from 23000 to $45000 \mathrm{~K}$ ). The SWS spectra are decomposed coherently in our sample into Lorentz profiles and a broadband continuum. We find that the individual profiles of the main AIBs at 3.3, 6.2, 8.6 and $11.3 \mu \mathrm{m}$ are well represented with at most two Lorentzians. The $7.7 \mu \mathrm{m}$-AIB has a more complex shape and requires at least three Lorentz profiles. Furthermore, we show that the positions and widths of these AIBs are remarkably stable (within a few $\mathrm{cm}^{-1}$ ) confirming, at higher spectral resolution, the results from ISOCAM-CVF and ISOPHOT-S. This spectral decomposition with a small number of Lorentz profiles implicitly assumes that most of the observed bandwidth arises from a few, large carriers. Boulanger et al. (1998b) recently proposed that the AIBs are the intrinsic profiles of resonances in small carbon clusters. This interpretation can be tested by comparing the AIB profile parameters (band position and width) given in this work to laboratory data on relevant species when it becomes available.

Taking advantage of our decomposition, we extract the profiles of individual AIBs from the data and compare them to a state-of-the-art model of Polycyclic Aromatic Hydrocarbon (PAH) cation emission. In this model, the position and width of the AIBs are rather explained by a redshift and a broadening of the PAH vibrational bands as the temperature of the molecule increases (Joblin et al. 1995). In this context, the present similarity of the AIB profiles requires that the PAH temperature distribution remains roughly the same whatever the radiation field hardness. Deriving the temperature distribution of interstellar PAHs, we show that its hot tail, which controls the AIB spectrum, sensitively depends on $N_{\min }$ (the number of C-atoms in the smallest PAH) and $T_{\text {eff }}$. Comparing the observed profiles of the individual AIBs to our model results, we can match all the AIB profiles (except the $8.6 \mu \mathrm{m}$-AIB profile) if $N_{\text {min }}$ is increased with $T_{\text {eff }}$. This increase is naturally explained in a picture where small PAHs are more efficiently photodissociated in harsher radiation fields. The observed $8.6 \mu \mathrm{m}$-profile, both intensity and width, is not explained by our model.

We then discuss our results in the broader context of ISO observations of fainter interstellar regions where PAHs are expected to be in neutral form.
\end{abstract}

Key words. infrared: ISM: lines and bands - ISM: dust, extinction - ISM: molecules

\section{Introduction}

The family of infrared features at $3.3,6.2,7.7,8.6,11.3$ and $12.7 \mu \mathrm{m}$ has been observed towards a large number of sightlines in the Galaxy and in other galaxies since

Send offprint requests to: L. Verstraete, e-mail: Laurent.Verstraete@ias.u-psud.fr

* Based on observations with ISO, an ESA project with instruments funded by ESA Member States (especially the PI countries: France, Germany, the Netherlands and the UK) and with the participation of ISAS and NASA. the nineteen seventies. Early on, it was recognized that these bands correspond to vibrational modes in carbonaceous aromatic systems (Duley \& Williams 1981; Léger \& Puget 1984; Allamandola et al. 1985). These dust bands are therefore dubbed the Aromatic Infrared Bands (hereafter AIBs). The bands at 3.3, 8.6, 11.3 and $12.7 \mu \mathrm{m}$ stem from vibrational modes of the aromatic $\mathrm{C}-\mathrm{H}$ bond; the remaining bands are ascribed to vibrations of the aromatic C-C bonds. The Infrared Space Observatory (ISO, Kessler et al. 1996) mission has provided us with an unprecedented wealth of data in this context. 
Comparative studies of the AIBs in a wide variety of environments have been made possible by the high sensitivity of the ISO camera (ISOCAM, Cesarsky C. et al. 1996) with its circular variable filter and of the ISOPHOT-S spectrophotometer (Lemke et al. 1996), which both have low spectral resolutions $(\lambda / \Delta \lambda \sim$ 40 and 90 respectively: Boulanger et al. 1996, 1998a, 1998b; Cesarsky et al. 1996a, 1996b, 2000a, 2000b; Crété et al. 1999; Klein et al. 1999; Laureijs et al. 1996; Mattila et al. 1996; Persi et al. 1999; Uchida et al. 1998, 2000). The AIB profiles as seen in these data are very similar (in position and width) over a range of objects where the stellar radiation field and effective temperature vary greatly ( 1 to $10^{4}$ times the standard interstellar radiation field, $T_{\text {eff }}=11000$ to $50000 \mathrm{~K}$ ). The profile invariance as well as the large width of the AIBs lead Boulanger et al. (1998b) to conclude that the carriers of these bands are large aromatic systems containing more than $50 \mathrm{C}$-atoms.

The Short Wavelength Spectrometer (SWS, de Graauw et al. 1996) onboard ISO, less sensitive than ISOCAM but with higher spectral resolution $(\lambda / \Delta \lambda=1000)$ and broader wavelength coverage $\left(2.4^{-}\right.$ $45 \mu \mathrm{m})$, brings a better view of the interstellar AIBs in the brightest regions. Such detailed data have the ability to constrain the nature and physical state of the band carriers (Beintema et al. 1996; Molster et al. 1996; Roelfsema et al. 1996; Verstraete et al. 1996; Moutou et al. 2000; van Kerckhoven et al. 2000; Hony et al. 2000). In the first part of this paper, we decompose the AIB spectrum into Lorentz profiles and a broadband continuum in order to characterize (band position and width) each individual AIB and to compare them between objects. In the second part of the paper, we compare these new observations with a model considering free-flying aromatic molecules (Polycyclic Aromatic Hydrocarbons or PAHs) as the origin of the AIBs. Indeed, the presence of AIBs in the low-excitation diffuse interstellar medium (Boulanger et al. 1996; Mattila et al. 1996; Onaka et al. 1996) requires the existence of free-flying PAHs or small grains excited by starlight; furthermore, these PAHs or grains must be small enough to undergo strong temperature fluctuations leading to emission of the AIBs in the near-infrared (Sellgren 1984). In this emission mechanism, the shape of the emergent AIB spectrum only depends on the radiation field hardness (or $T_{\text {eff }}$, see Sect. 2) and not on the flux of stellar photons (parameterized as $\chi$ in Sect. 2).

In Sect. 2, we present the SWS spectra. The observed AIB profiles are characterized in Sect. 3 . These results are compared to the predictions of the PAH model in Sect. 4. We summarize and discuss the significance of our results in Sect. 5 .

\section{Observations and physical conditions}

SWS has observed the AIB spectrum along a number of interstellar sightlines covering a wide range of excitation conditions. We discuss here three spectra which sample well the radiation field sequence covered by the SWS observations. The lines of sight selected all correspond to interface conditions, i.e., regions where fresh molecular material is directly exposed to the stellar light. At interfaces, the AIB emission is usually strong (probably because of an enhanced PAH abundance, Bernard et al. 1993): this is why we selected such regions to carry out the present study.

At the low excitation end, we have the reflection nebula NGC 2023 (TDT $=65602309$, SWS01 speed 3). This spectrum has already been presented in Moutou et al. (1999). SWS looked at a filament, $60^{\prime \prime}$ south of the central star (central star: RA $=5^{\mathrm{h}} 41^{\mathrm{m}} 38.3^{\mathrm{s}}, \mathrm{DEC}=$ $-2^{\circ} 16^{\prime} 32.6^{\prime \prime}$ ), which is bright in fluorescent $\mathrm{H}_{2}$ emission (Field et al. 1998). At the high excitation end, we present here a spectrum of the M17-SW photodissociation interface $\left(\mathrm{RA}=18^{\mathrm{h}} 20^{\mathrm{m}} 22.1^{\mathrm{s}}, \mathrm{DEC}=-16^{\circ} 12^{\prime} 41.3^{\prime \prime}\right.$; TDT $=32900866$, SWS01 speed 4): this is position number 6 of the data presented in Verstraete et al. (1996). Finally, we also have the Orion Bar $\left(\mathrm{RA}=5^{\mathrm{h}} 35^{\mathrm{m}} 20.3^{\mathrm{s}}\right.$, $\mathrm{DEC}=-5^{\circ} 25^{\prime} 20^{\prime \prime} ;$ TDT $=69501806$, SWS01 speed 4$)$ at the position of the peak of fluorescent $\mathrm{H}_{2}$ emission (van der Werf et al. 1996). All positions given above are in J2000.

The data reduction was undertaken with the SWS-IA3 environment running at the Institut d'Astrophysique Spatiale, Orsay. The spectrum of NGC 2023 lacks a small range around $4 \mu \mathrm{m}$ because of bad dark current measurements. The flux calibration files CAL-G version 030 have been used. For the beam sizes, we took the values recently determined by Salama (2000). This assumes that the source completely fills the beam. To check this assumption, we compared our SWS spectra of M17-SW and of the Orion Bar to CAM-CVF data (Cesarsky et al. 1996a,b; Cesarsky et al. 2000b): the continuum fluxes (per solid angle) of the two instruments were found to agree within $20 \%$. In the case of NGC 2023, the emission seen in the ISOCAM-map of Abergel et al. (2000, in preparation) looks homogeneous at the position of our SWS spectrum. The 6 arcsecond pixels of ISOCAM are much smaller than the SWS field of view, and since the ISOCAM image of each of our sources is smooth in the region observed by SWS, we can safely say that our sources uniformly fill the SWS beam. This statement only holds over the $5-16 \mu \mathrm{m}$ wavelength range. In fact, to assure continuity in our spectra, we had to deviate from the Salama SWS beam sizes above $27 \mu \mathrm{m}$ (the spectral bands $3 \mathrm{E}$ and 4 of the SWS, see de Graauw et al. 1996).

The resulting 2.4-25 $\mu \mathrm{m}$ spectra are shown in Fig. 1. As we will see below, this reduced spectral range amply suffices in our discussion of the AIB profiles. The NGC 2023 spectrum has a resolving power $\lambda / \Delta \lambda=200$ and those of M17-SW and of the Orion Bar have $\lambda / \Delta \lambda=$ 500 . The physical conditions prevailing in each interstellar region are summarized in Table 1 . We assumed that the radiation field from the exciting star is a blackbody characterized by an effective temperature. In this table, we give the effective temperature of the exciting star $\left(T_{\text {eff }}\right)$, the dilution factor for the blackbody $\left(W_{\text {dil }}\right)$, the flux 


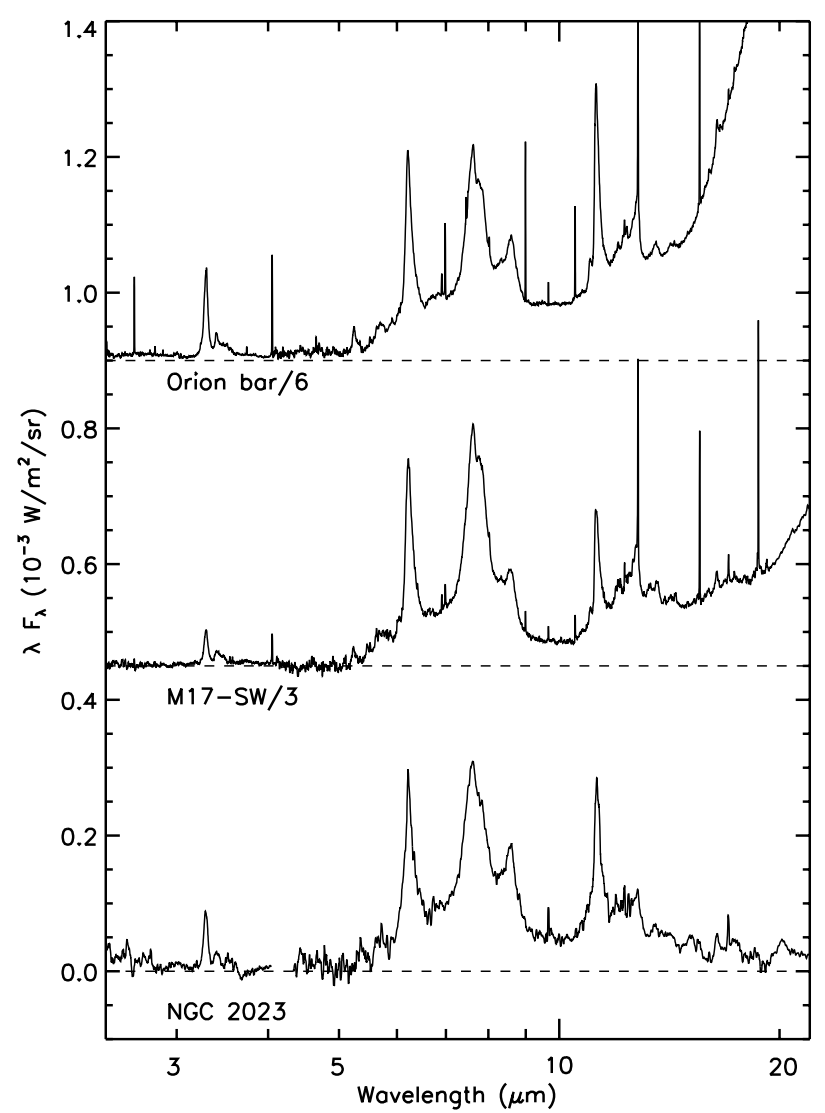

Fig. 1. The SWS 2.4-25 $\mu \mathrm{m}$ spectra of the AIBs towards various interstellar sources (see text). The spectra have been normalized to the $6.2 \mu \mathrm{m}$-feature of NGC 2023; the scaling factors are given in the figure, e.g., M17-SW/3 means that the flux of M17-SW has been divided by 3. A positive constant has been added to all spectra except that of NGC 2023 to ease comparison. The zero flux level of each spectrum is indicated by the dashed line. The resolving power $(\lambda / \Delta \lambda)$ is 200 for NGC 2023 and 500 for M17-SW and the Orion Bar. The narrow, unresolved lines are due to $\operatorname{Br} \beta 2.63 \mu \mathrm{m}, \operatorname{Br} \alpha 4.06 \mu \mathrm{m}$, forbidden ionic lines ([ArII] $6.98 \mu \mathrm{m},[$ ArIII] $8.99 \mu \mathrm{m}$, [SIV] $10.5 \mu \mathrm{m}$, [NeII] $12.8 \mu \mathrm{m},[\mathrm{NeIII}] 15.5 \mu \mathrm{m}$ and [SIII] $18.7 \mu \mathrm{m})$ and $\mathrm{H}_{2}$ pure rotational lines $(0-0 \mathrm{~S}(5) 6.91 \mu \mathrm{m}, 0-0 \mathrm{~S}(4) 8.02 \mu \mathrm{m}$, 0-0 S(3) $9.66 \mu \mathrm{m}, 0-0 \mathrm{~S}(2) 12.3 \mu \mathrm{m}$ and 0-0 $\mathrm{S}(1) 17.0 \mu \mathrm{m})$.

scaling factor $(\chi)$ in units of the Habing field (at $\lambda=$ $1000 \AA$ ) and $d_{\star}$ the distance of our line of sight from the central star (we assumed $d_{\star}$ to be the projected distance on the sky in all cases). In the case of M17-SW, several stars excite the regions we observed: in Table 1 we give effective values $\left(T_{\text {eff }}, R_{\star}\right)$ that reproduce well the sum of all radiation fields. Our excitation sequence thus goes from NGC 2023 (cool star) to M17-SW (hot star).

\section{The observed AIB profiles}

The continuum below the AIBs shows a clear evolution from NGC 2023 to the Orion Bar (see Fig. 1): continuous emission of hot, small (radii of a few 10 to $100 \AA$, Désert et al. 1990) grains between 10 and $20 \mu \mathrm{m}$ is prominent in M17-SW and the Orion Bar whereas this is completely absent in NGC 2023. This is a consequence of the stronger
Table 1. Physical conditions for the AIB spectra - see text.

\begin{tabular}{cccc}
\hline Line of sight & NGC 2023 & Orion Bar & M17-SW \\
\hline \hline$T_{\text {eff }}\left(10^{4} \mathrm{~K}\right)$ & $2.3^{a}$ & $3.7^{c}$ & $4.5^{e}$ \\
$R_{\star}\left(R_{\odot}\right)$ & $3.2^{a, b}$ & $8.5^{b}$ & 12 \\
$d_{\star}(\mathrm{pc})$ & 0.14 & 0.24 & 1.10 \\
$W_{\text {dil }} / 10^{-13}$ & 2.66 & 6.38 & 0.96 \\
$\chi / 10^{3}$ & 1.2 & 42 & 12.5 \\
\hline
\end{tabular}

The distances to NGC 2023, M17-SW and the Orion Bar were taken to be $480 \mathrm{pc}^{a}, 2.2 \mathrm{kpc}^{e}$ and $460 \mathrm{pc}^{d}$ respectively. References: (a) Buss et al. (1994), (b) Lang (1991), (c) Rubin et al. (1991), (d) van der Werf et al. (1996), (e) Felli et al. (1984)

flux and harder radiation field in the Orion Bar which heats up these small grains.

The 6.2 and $7.7 \mu \mathrm{m}$ bands do not vary much with respect to each other whereas they do relative to the 3.3, 8.6 and $11.3 \mu \mathrm{m}$ AIBs. Such variations cannot be interpreted as the result of different emission temperature distributions of PAHs (obtained with different size distributions and/or radiation field effective temperature). In fact, the $3.3 \mu \mathrm{m}$-band is dominated by the emission of small (hot) PAHs while the $11.3 \mu \mathrm{m}$-band is contributed to by larger (cold) PAHs (Schutte et al. 1993): thus, changing the PAH temperature distribution cannot explain why the $11.3 \mu \mathrm{m}$-band varies along with the $3.3 \mu \mathrm{m}$-band. Instead, modifications of the physical state of PAHs have to be invoked. Theoretical (de Frees et al. 1993; Pauzat et al. 1995, 1997; Langhoff 1996) and laboratory (Szczepanski \& Vala 1993; Hudgins et al. 1994; Hudgins \& Allamandola 1995; Hudgins \& Sandford 1998) studies showed that ionized species have stronger C-C bands. On the other hand, dehydrogenated PAHs have weaker $\mathrm{C}-\mathrm{H}$ bands (Pauzat et al. 1995, 1997). As we show in Sect. 4.4.1, the weaker $\mathrm{C}-\mathrm{H}$ band emission in M17-SW can be explained if the hydrogenation fraction is significantly reduced.

Moreover, the AIB profiles show little spectral substructure even though most AIBs are fully resolved in our SWS data (the resolving power is $\lambda / \Delta \lambda=200$ to 500). A direct comparison of the spectra displayed in Fig. 1 shows that the position and width of the major, simple AIBs (at 3.3, 6.2, 8.6 and $11.3 \mu \mathrm{m}$ ) are roughly the same. The decomposition of the spectra we discuss below primarily aims at quantifying this comparison and also at disentangling in a systematic way the profile of a given AIB from the other bands, as well as from the underlying continuum due to hot small grains.

Boulanger et al. (1998b) showed that the AIBs in CAM-CVF data can be decomposed into Lorentz profiles and a linear underlying continuum. It must be emphasized, however, that the AIBs are barely resolved in the CAM-CVF data (in particular the 6.2, 8.6 and $11.3 \mu \mathrm{m}$ bands, see Table 2 in Boulanger et al. 1998b and Fig. 3 of Cesarsky et al. 2000). The SWS data at high spectral resolution presented here do not suffer from this limitation. The use of a Lorentzian band shape implicitly assumes that the AIB profiles arise from the intrinsic width 
of molecular transitions and/or resonances in small solid particles (e.g., Bohren \& Huffman 1983).

In the current paradigm, the AIBs result from the superposition of many vibrational bands produced by a population of interstellar PAHs with a wide range of sizes (molecules containing a few tens to a few hundred carbon atoms, Désert et al. 1990; Schutte et al. 1993). In this respect, laboratory and theoretical studies on small $\mathrm{PAH}$ species teach us that the band shapes (position and width) of vibrational transitions (i) depend on the temperature of the molecule (Joblin et al. 1995) and, (ii) vary from one species to another (in particular, the position of the vibrational bands depends on the size and symmetry of the molecule: Szczepanski \& Vala 1993; Hudgins \& Allamandola 1995 and 1998; Joblin et al. 1995; Langhoff 1996). The observed AIBs may actually result from a combination of these two effects. Elaborating on these studies, variability in the AIBs (changing band ratios, presence of substructure in the band profiles) from different interstellar sightlines is predicted as a consequence of a changing $\mathrm{PAH}$ population and/or different exciting radiation fields. Specifically, the position and width of individual bands are expected to vary by a few to several tens of $\mathrm{cm}^{-1}$ from one species to another and/or as a result of different emission temperatures. Some changes in the AIB profiles have been observed towards $\mathrm{H}$ II regions and reflection nebulae (Roelfsema et al. 1996; Verstraete et al. 1996; Cesarsky et al. 2000a; Uchida et al. 2000; Peeters et al. 2000 in preparation). The case of the general (bright) interstellar medium is covered below with a quantitative comparison of the AIB profiles.

In this work, rather than establish the "final" AIB profile parameters, we aim at comparing on the same footing the individual AIB profiles under different excitation conditions. We have therefore decomposed the present spectra into Lorentz profiles and a modified blackbody as underlying continuum. We restricted ourselves to the minimum number of Lorentz profiles required in order to produce a reasonable overall fit and a good representation of every individual AIB.

We used a classical gradient-expansion algorithm with analytical partial derivatives and performed the fit in the wavenumber space $\left(x=1 / \lambda\right.$ in $\left.\mathrm{cm}^{-1}\right)$ over the $2.4-25 \mu \mathrm{m}$ wavelength range. In addition to the Lorentz profiles, the underlying continuum is fitted simultaneously. For the latter, we took a modified blackbody with an emissivity law proportional to $x$, the temperature and peak brightness of which were the free parameters. The same set of fit parameters was used for all objects. Such a set was first fixed on the M17-SW spectrum which has a high signal-to-noise ratio and a good feature-to-continuum contrast. To fit the 2.4-25 $\mu \mathrm{m}$ spectrum, twenty Lorentz profiles were necessary. Then, the parameter values of the Lorentz profiles and of the continuum in M17-SW were used as input to fit the other AIB spectra: very good fits were obtained by first relaxing the Lorentz amplitudes and blackbody continuum parameters, suggesting a complete and robust decomposition of the AIB spectrum. After adjusting the amplitudes, the profile (position and width) and continuum parameters were fine-tuned, simultaneously, over restricted spectral ranges. The centroids, widths and amplitudes of the Lorentzian fits to the main AIBs are given in Table 2. Our fit to the $5-25 \mu \mathrm{m}$-AIB spectrum is shown in Fig. 2 . The blackbody component of this decomposition is consistent with the emission of warm dust in the mid-infrared and in particular its exponential decay (the Wien tail of the blackbody). The observed strong variability of the $20 \mu \mathrm{m}-$ continuum flux (very weak in NGC 2023 whereas strong in M17-SW and the Orion Bar) then reflects the varying temperature of the warm dust component. As can be seen in Fig. 2, the underlying continuum contributes little below the AIBs. On the other hand, additional, colder blackbody, type continua are required to fit the full SWS spectra out to $45 \mu \mathrm{m}$.

We note that broad bands are required at about 1000 and $1450 \mathrm{~cm}^{-1}$ in order to explain the continuum between the AIBs. These bands may not be associated with the AIBs but, for simplicity, we assumed their profiles to be Lorentzian. Their parameters are not well constrained in our decomposition: the sole requirement is that the corresponding profiles are broad enough to reproduce the smooth continuum observed in these spectral regions. The fitted profiles of the neighbouring AIBs are somewhat sensitive to the widths adopted for the 1000 and $1450 \mathrm{~cm}^{-1}$ bands: for instance, if the full width at half maximum $(F W H M)$ of the $1450 \mathrm{~cm}^{-1}$ band is increased from 200 to $300 \mathrm{~cm}^{-1}$, the $6.2 \mu \mathrm{m}$-band has its $F W H M$ reduced by $2.5 \mathrm{~cm}^{-1}$ and its position redshifted by $0.8 \mathrm{~cm}^{-1}$. In order to coherently compare the AIB profiles, we have fixed the width of these broad bands: namely, $F W H M=300 \mathrm{~cm}^{-1}$ for the $1000 \mathrm{~cm}^{-1}$-band and $F W H M=200 \mathrm{~cm}^{-1}$ for the $1450 \mathrm{~cm}^{-1}$-band. At this stage, we can point out that combinations of PAH vibrational modes have been predicted to accumulate between 1000 and $2000 \mathrm{~cm}^{-1}$ in a broad structure (Bernard et al. 1989).

Also noteworthy is that two Lorentzians are required to correctly reproduce the red wing asymmetry of the $1609 \mathrm{~cm}^{-1}(6.2 \mu \mathrm{m})$ and $890 \mathrm{~cm}^{-1}(11.3 \mu \mathrm{m})$ bands: these components are labelled "core" and "red wing" in Table 2. The feature centered around $1300 \mathrm{~cm}^{-1}$ (the classical "7.7 $\mu$ m-band") shows 3 sub-peaks at about 1273, 1310 and $1328 \mathrm{~cm}^{-1}$. In the following, we will call the narrow $1310 \mathrm{~cm}^{-1}$ peak the "7.6 $\mu \mathrm{m}$-band" while the broader 1273 and $1328 \mathrm{~cm}^{-1}$ components will be dubbed the "7.8" and "7.5 $\mu \mathrm{m}$-bands" respectively. The observed $1310 \mathrm{~cm}^{-1}$-feature has a narrow core and a broad blue wing which demands another component at $1328 \mathrm{~cm}^{-1}$. A Lorentzian is also required around $1204 \mathrm{~cm}^{-1}\left(F W H M \sim 70 \mathrm{~cm}^{-1}\right)$ to fill the gap between the 7.8 and the $8.6 \mu \mathrm{m}$-features. In the case of Orion this band shifts to $1209 \mathrm{~cm}^{-1}$ in order to reproduce the pronounced and extended blue wing of the $8.6 \mu \mathrm{m}$-band.

In Fig. 3 we show the profile and fit of the $3.3 \mu \mathrm{m}$ band. The band shape is also Lorentzian and the continuum has the same functional form as in the $5-25 \mu \mathrm{m}$ region. In 
Table 2. Fit parameters of the AIB Lorentz profiles.

\begin{tabular}{|c|c|c|c|}
\hline AIB & NGC 2023 & M17-SW & Orion Bar \\
\hline \multirow[t]{3}{*}{$11.3 \mu \mathrm{m}$} & $888.6^{1}$ & 889.9 & 889.7 \\
\hline & $20.8^{2}$ & 17.8 & 14.2 \\
\hline & $877^{3}$ & 2292 & 7241 \\
\hline \multirow[t]{3}{*}{ Core } & 889.3 & 890.2 & 889.9 \\
\hline & 13.1 & 10.1 & 10.9 \\
\hline & 564 & 1644 & 5928 \\
\hline \multirow[t]{3}{*}{ Red wing } & 881.9 & 880.3 & 880.5 \\
\hline & 29.6 & 30.4 & 30.0 \\
\hline & 384 & 915 & 1820 \\
\hline \multirow[t]{3}{*}{$8.6 \mu \mathrm{m}$} & 1164.0 & 1163.8 & 1161.5 \\
\hline & 49.3 & 44.6 & 48.0 \\
\hline & 355 & 780 & 1957 \\
\hline \multirow[t]{3}{*}{$7.8 \mu \mathrm{m}$} & 1274.2 & 1273.5 & 1275.1 \\
\hline & 70.2 & 67.6 & 54.2 \\
\hline & 392 & 1494 & 2417 \\
\hline \multirow[t]{3}{*}{$7.6 \mu \mathrm{m}$} & 1309.9 & 1312.6 & 1311.3 \\
\hline & 22.9 & 28.6 & 25.6 \\
\hline & 130 & 912 & 1760 \\
\hline \multirow{3}{*}{$7.5 \mu \mathrm{m}$} & 1328.1 & 1327.8 & 1329.7 \\
\hline & 68.2 & 74.5 & 55.9 \\
\hline & 466 & 1074 & 2022 \\
\hline \multirow[t]{3}{*}{$6.2 \mu \mathrm{m}$} & 1608.1 & 1607.7 & 1609.7 \\
\hline & 48.6 & 42.6 & 38.9 \\
\hline & 550 & 1748 & 3428 \\
\hline \multirow[t]{3}{*}{ Core } & 1608.5 & 1608.6 & 1610.5 \\
\hline & 17.1 & 30.0 & 25.5 \\
\hline & 224 & 1376 & 2369 \\
\hline \multirow[t]{3}{*}{ Red wing } & 1600.5 & 1586.2 & 1594.9 \\
\hline & 80.0 & 64.4 & 64.8 \\
\hline & 338 & 546 & 1291 \\
\hline \multirow[t]{3}{*}{$3.3 \mu \mathrm{m}$} & 3041.8 & 3039.1 & 3040.0 \\
\hline & 43.0 & 38.8 & 40.4 \\
\hline & 98 & 171 & 834 \\
\hline
\end{tabular}

${ }^{1}$ center in $\mathrm{cm}^{-1}\left( \pm 0.8 \mathrm{~cm}^{-1}\right.$, see text).

2 width in $\mathrm{cm}^{-1}\left( \pm 2 \mathrm{~cm}^{-1}\right)$.

${ }^{3}$ amplitude in $\mathrm{MJy} / \mathrm{sr}$.

other words, we fitted the 2.4-25 $\mu \mathrm{m}$ continuum with a single blackbody component.

The decomposition presented above is not unique: we also tried to use a multicomponent power-law continuum $\left(A / x^{3}+B / x^{2}+C / x+D\right.$ with $x=1 / \lambda$ and where $A, B, C$ and $D$ are constant parameters) below the AIBs from 2.4 to $16 \mu \mathrm{m}$. Such a continuum gives a notable contribution below the AIBs, in particular, it replaces completely the $1000 \mathrm{~cm}^{-1}$ broad band. However, a multicomponent power-law continuum has no straightforward physical interpretation and it cannot describe the strong rise of the spectrum beyond $16 \mu \mathrm{m}$ observed in M17-SW and the Orion Bar. Nor can it accommodate the weak $20 \mu$ m-flux in NGC 2023. In any case, comparing the fits obtained with the two types of continua (power-law and blackbody), we found the positions and widths of the main AIBs characterized in Table 2 to vary by less than 1.6 and $4 \mathrm{~cm}^{-1}$ respectively in a given object. Finally, using gaussian band shapes for the AIBs, we got as good a decomposition (see also Boulanger et al. 1998b): yet the

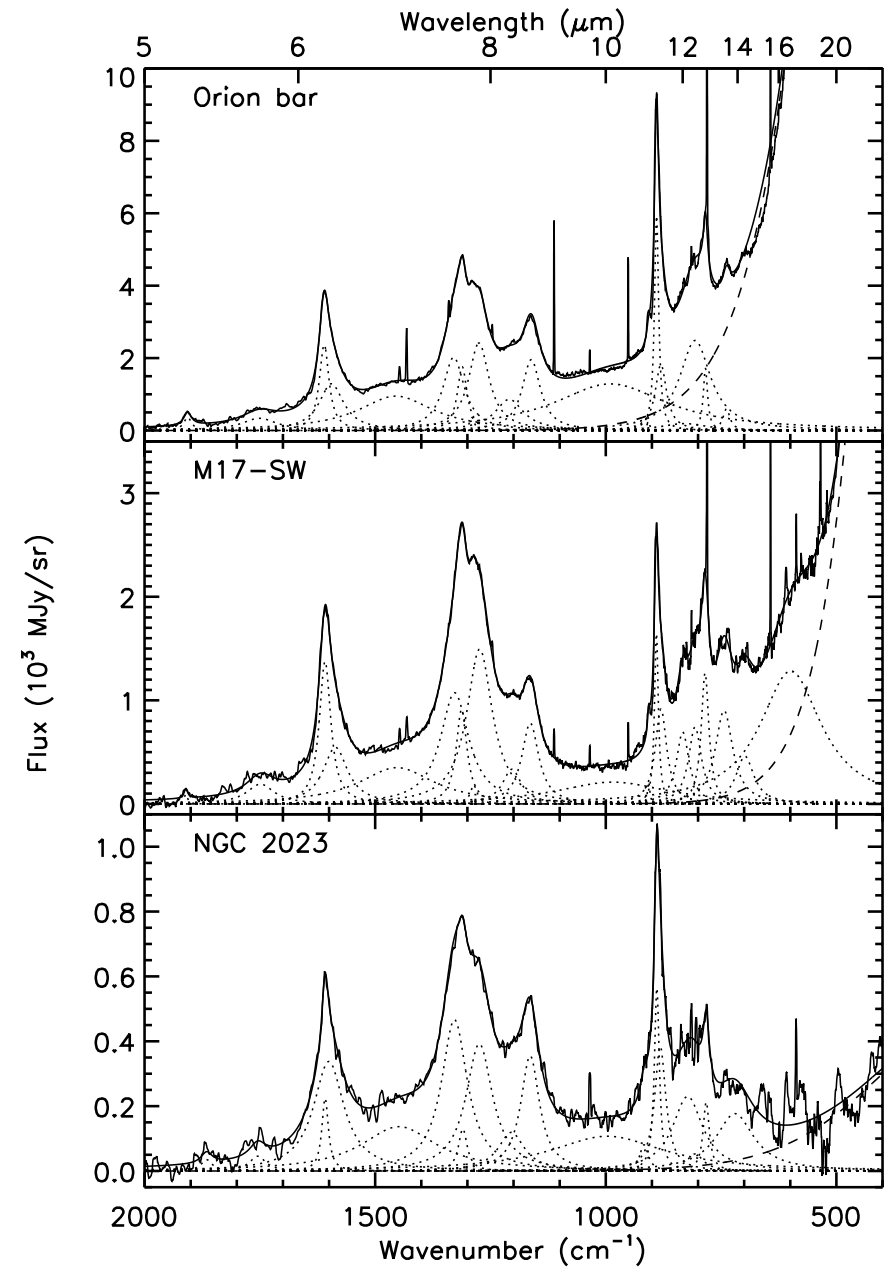

Fig. 2. The decomposition of the AIB spectra between 5 and $25 \mu \mathrm{m}$. The resolving power $(\lambda / \Delta \lambda)$ of the data is 200 for NGC 2023 and 500 for M17-SW and the Orion Bar. Each Lorentz component is represented by a dotted line, the underlying continuum by a dashed line. The full fit is the solid line.

continuum under the AIBs was stronger and more structured because of the weaker profile wings. We believe that the fit quality does not preclude any band profile: in fact, the SWS AIB spectrum is so rich that there are always enough parameters in the fit to accomodate any choice of band profile.

We have thus determined in a coherent way the position and width of the strong, well-delineated AIBs at $3.3,6.2,7.6,8.6$ and $11.3 \mu \mathrm{m}$ with an accuracy of 0.8 and $2 \mathrm{~cm}^{-1}$ respectively. Inspection of Table 2 shows that there are significant variations in the width of the 6.2 and $11.3 \mu \mathrm{m}$-bands (9.7 and $6.6 \mathrm{~cm}^{-1}$ respectively) as well as in the position of the 3.3 and $7.6 \mu \mathrm{m}$-bands $\left(2.7 \mathrm{~cm}^{-1}\right.$ in both cases). However, all these variations come within the accuracy range given above when the values of NGC 2023 are excluded. In this latter object, the poorer signal-tonoise ratio and resolving power (see Fig. 1 and Figs. 8 to 11) has degraded the band profiles: this is probably why some AIB parameters are singular in NGC 2023. 


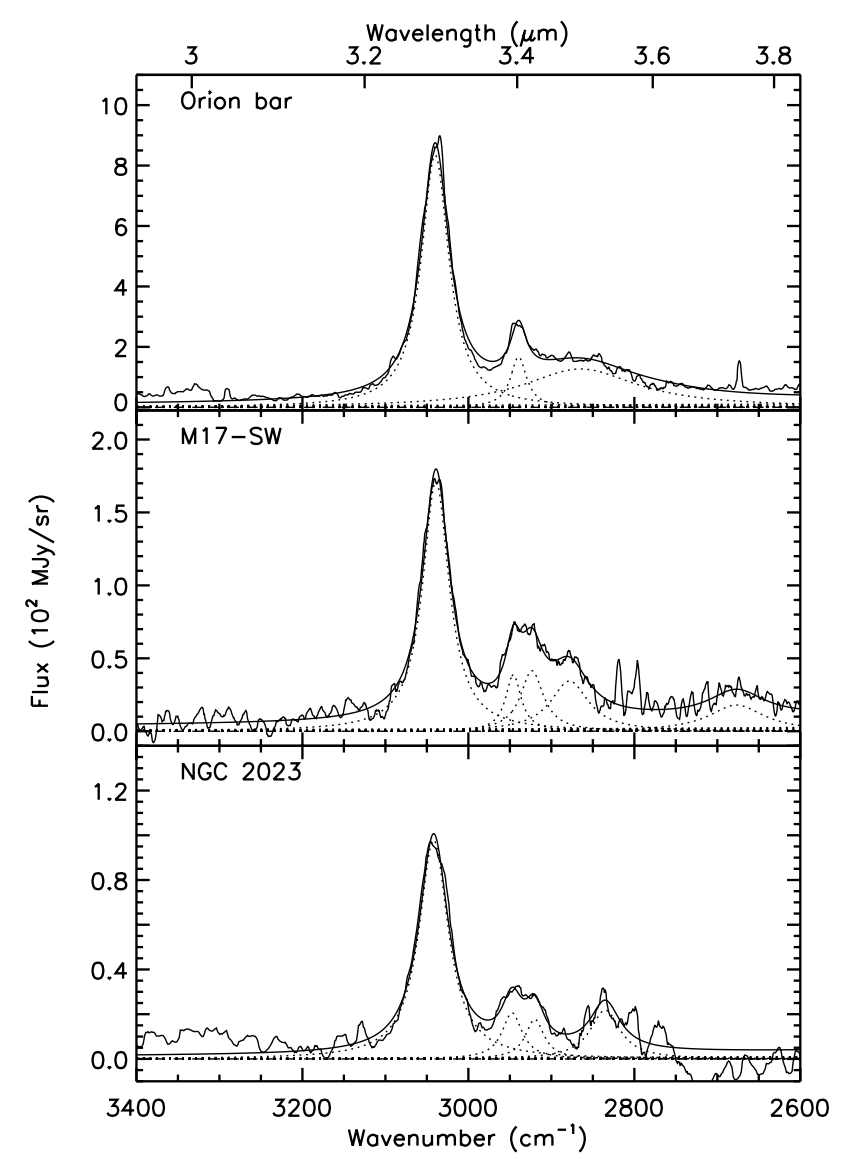

Fig. 3. Same as Fig. 2 in the region of the $3.3 \mu \mathrm{m}$ AIB. Note that the continuum has the same functional form than that already seen in Fig. 2. In the Orion Bar spectrum, the Pf $\delta$ hydrogen recombination line clearly visible on the top of the $3.3 \mu \mathrm{m}$ band was masked to perform the fit. The Pf $\gamma$ at $3.74 \mu \mathrm{m}$ and Pf $\epsilon$ at $3.04 \mu \mathrm{m}$ lines can also be seen in emission in the Orion Bar spectrum.

On the other hand, we find that the position of the $8.6 \mu \mathrm{m}$ band varies by $2.5 \mathrm{~cm}^{-1}$ (Table 2) and that most of this variation is due to a redshift in the Orion Bar spectrum. As noted above, the $8.6 \mu \mathrm{m}$-band in this object has an extended blue wing: this spectral change is related to the redshift of the band itself. This result may point to the more profound modifications seen in this spectral range by Roelfsema et al. (1996) and Verstraete et al. (1996) towards more excited regions.

From the above discussion, we conclude that most AIB profiles (except the $8.6 \mu \mathrm{m}$-AIB in the Orion Bar) do not vary within the accuracy of our spectral decomposition. At $3.3 \mu \mathrm{m}$, a similar result was already obtained by Tokunaga et al. (1991) who compared the band profile of planetary nebulae and $\mathrm{H}$ II regions (their Type 1 profile) with excitation conditions $\left(T_{\text {eff }}\right.$ and $\left.\chi\right)$ similar to that of our sample (see Table 1). Roche et al. (1996) confirmed this result on a larger, lower-excitation sample of planetary nebulae. Similarly, Witteborn et al. (1989) showed that the $11.3 \mu \mathrm{m}$-band profile is rather stable. Furthermore, the observed smoothness and invariance of the AIBs across a wide range of excitation conditions appears difficult to reconcile with the variability expected from laboratory results.

Having performed this mathematical parameterization of the SWS spectra, we are now able to extract the individual AIB profiles and to compare them in different objects and to model predictions. Yet, what is the physical meaning of this spectral decomposition? We have chosen Lorentzian band shapes and we represented most AIBs (except the $7.7 \mu \mathrm{m}$ ) with one or two Lorentz profiles: this implicitly assumes that the AIBs arise from a few vibrational bands common to many PAHs and that most of the bandwidth arises from a single carrier. The invariance and smoothness of the AIBs is then naturally explained (Boulanger et al. 1998b). To verify this interpretation of the AIBs, the band parameters of Table 2 can be compared directly to laboratory or theoretical studies on relevant PAHs (large molecules containing more than 50 C-atoms as argued by Boulanger et al. 1998b). But there are other ways to look at the interstellar AIB spectrum which do not result in Lorentzian bandshapes. For instance, simulations of astronomical spectra based on recent laboratory studies of small PAHs (Allamandola et al. 1999) have shown that the AIB spectrum may be decomposed by assigning a multiplicity of (species dependent) vibrational bands to each AIB. Another possibility to interpret the AIB spectrum is based on the laboratory work of Joblin et al. (1995) which shows that the vibrational bands of PAHs are broadened and redshifted as the temperature of the molecule is raised. In this picture, the profile of a vibrational band from a single molecule with a given internal energy has a Lorentz shape (the intrinsic profile) which only depends on the temperature of the molecule (and not on the species). The observed AIBs are then interpreted as the superposition of many Lorentz profiles corresponding to all the possible temperatures reached by a population of PAHs.

In the next section, devoted to modelling, we adopt and detail this latter view of the AIB spectrum. In this context, using the relationship between bandwidth and temperature established by Joblin et al. (1995), we note that the width of the observed $3.3 \mu \mathrm{m}$-band $\left(40 \mathrm{~cm}^{-1}\right)$ is well explained if the emitting $\mathrm{PAH}$ has a temperature of about $1000 \mathrm{~K}$. Such emission temperatures are in good agreement with what is expected for interstellar PAHs emitting during temperature fluctuations (see Sect. 4). Using the present spectral decomposition of the SWS data, we can now extract the individual AIB profiles and compare them to the predictions of a PAH emission model that uses the best available laboratory data on PAHs.

\section{Probing the PAH model}

To first order, the AIB profiles essentially remain identical while the exciting radiation hardens considerably: $T_{\text {eff }}=23000 \mathrm{~K}$ in NGC 2023 to $45000 \mathrm{~K}$ in M17-SW corresponding to the mean energy of the photons absorbed by a PAH of 6 and $9 \mathrm{eV}$ respectively. Such contrasted internal energies imply differences in the emission temperature 
of a few $100 \mathrm{~K}$ for a given molecular size. With this temperature change, band position shifts and band broadening of at least $10 \mathrm{~cm}^{-1}$ are expected (Joblin et al. 1995). How can then the interstellar AIB profiles be so stable? One possibility is that some process keeps the temperature distribution of interstellar PAHs essentially the same.

To investigate this issue, we have computed the temperature distribution and associated infrared (IR) emission of an interstellar PAH population using a modified version of the model described in Pech et al. (2000 hereafter PJB) where we study in detail the temperature fluctuations and the temperature distribution of PAHs. Improving on former work (Léger et al. 1989b; Schutte et al. 1993; Cook \& Saykally 1998), this model takes into account the full spectral distribution of the exciting radiation and implements the recent results of Joblin et al. (1995) on the temperature dependence of the band profiles of neutral PAHs. In addition, it must be emphasized that the PAH IR emission cross-section of the PJB model was derived from studies on PAH cations: indeed, we show in Sect. 4.4.1 that singly ionized PAHs reproduce better the astronomical AIB spectra confirming previous work (Langhoff 1996; Cook \& Saykally 1998; Allamandola et al. 1999; Hudgins \& Allamandola 1999). This observational requirement is supported by theoretical studies on the charge state of interstellar PAHs for physical conditions comparable to those of our sample of objects (Bakes \& Tielens 1994; Dartois \& d'Hendecourt 1997). We first briefly describe the equations giving the PAH cooling curve and emission, compute the temperature distribution of interstellar PAHs and then compare our model PAH emission spectrum to the data. For the sake of clarity, we limit the model-to-data comparison to NGC 2023 and M17-SW which are the extreme cases of our data sample and we focus on the well-defined 3.3, 6.2, 8.6 and $11.3 \mu \mathrm{m}$-bands. In order to emphasize the temperature fluctuations of PAHs and the resulting temperature distribution, we have written the model equations in terms of cross-sections rather than Einstein $A$-coefficients: this formulation is however strictly equivalent to that of PJB (see, e.g., Schutte et al. 1993, Eq. (15)).

\subsection{Emission by PAHs}

In the interstellar medium, a PAH containing $N_{\mathrm{C}}$ carbon atoms absorbs radiation from the surrounding stars at a rate:

$R_{\text {abs }}\left(\right.$ photons s $\left.{ }^{-1}\right)=\int_{0}^{\infty} \sigma_{\nu}^{a} \frac{F_{\nu}}{h \nu} \mathrm{d} \nu$

with $\sigma_{\nu}^{a}$ the visible-ultraviolet (UV) absorption crosssection of PAHs (Verstraete \& Léger 1992) and $F_{\nu}$ the incident stellar flux assumed to be a diluted blackbody with the dilution factor and effective temperature given in Table 1. The absorption cross-section is proportional to $N_{\mathrm{C}}$; it also has a cut-off (due to the electronic transitions) in the visible to near-IR range whose wavelength increases with $N_{\mathrm{C}}$ (Verstraete \& Léger 1992). The mean photon energy absorbed by a molecule is given by:

$E_{\mathrm{abs}}=\frac{P_{\mathrm{abs}}}{R_{\mathrm{abs}}} \quad$ with $\quad P_{\mathrm{abs}}=\int_{0}^{\infty} \sigma_{\nu}^{a} F_{\nu} \mathrm{d} \nu$.

$P_{\text {abs }}$ is the power absorbed per molecule. In the standard interstellar radiation field of Mathis et al. (1983), we find that a PAH with $N_{\mathrm{C}}=25$ or 500 carbon atoms absorbs $2.4 \times 10^{-27}$ or $4 \times 10^{-27}$ Watt per C-atom with $E_{\text {abs }}=$ 4.8 or $2.3 \mathrm{eV}$, respectively. The values for $N_{\mathrm{C}}=25$ are in good agreement with the laboratory results of Joblin et al. (1992) on small PAHs.

As shown by Léger et al. (1989a) and Schutte et al. (1993), the IR emission of a PAH is well treated within the thermal approximation and using Kirchhoff's law. In the following, we will discuss the complete spectrum emitted by PAHs in terms of the spectral energy distribution (SED) noted $S(\nu)$ which is equal to $\nu I_{\nu}$. The SED (in Watt) emitted by a single molecule containing $N_{\mathrm{C}}$ carbon atoms is thus:

$$
\begin{aligned}
& S\left(\nu^{\prime}, N_{\mathrm{C}}\right)=4 \pi R_{\mathrm{abs}} \\
& \quad \times \int_{0}^{\infty} \mathcal{P}_{\nu} \int_{0}^{t_{\mathrm{e}}} \nu^{\prime} \sigma_{\nu^{\prime}}^{\mathrm{e}} B_{\nu^{\prime}}\left(T_{\mathrm{c}}\left(t, \nu, N_{\mathrm{C}}\right)\right) \mathrm{d} t \mathrm{~d} \nu
\end{aligned}
$$

where

$\mathcal{P}_{\nu}=\frac{\sigma_{\nu}^{a}}{R_{\mathrm{abs}}} \times \frac{F_{\nu}}{h \nu}$

is the probability density for absorption of a photon of energy $h \nu, \sigma_{\nu^{\prime}}^{\mathrm{e}}$ the emission cross-section in the IR, $t_{\mathrm{e}}$ is the time at the end of cooling (see Sect. 4.2) and $T_{\mathrm{c}}\left(t, \nu, N_{\mathrm{C}}\right)$ is the cooling curve of the molecule. In the following, the frequency of photons emitted in the IR will be labelled $\nu^{\prime}$ and that of stellar photons absorbed in the visible-UV range will be written $\nu$.

The PAH IR emission cross-section is defined as in PJB. The band profiles have a Lorentzian shape and at $3.3,6.2,8.6$ and $11.3 \mu \mathrm{m}$, the band position and width follow temperature dependent laws. Each Lorentz profile is normalized to the peak value of the band cross-section, $\sigma_{\nu}$ and we assume that the integrated cross-section (or Einstein $A$-coefficient, see Schutte et al. 1993) of each band profile is conserved with temperature. As already noted above, the resulting IR cross-section describes the properties of singly-ionized PAHs. To match the observed AIB, the $8.6 \mu \mathrm{m}$-band had to be multiplied by 3 . Because the available laboratory results do not account for the complex shape of the observed $7.7 \mu \mathrm{m}$-band (see Sect. 3), we define an empirical $7.7 \mu \mathrm{m}$-band with a shape derived from the observations (profile centre at $1300 \mathrm{~cm}^{-1}$ and $F W H M=113 \mathrm{~cm}^{-1}$ from the present data) and with the (laboratory determined) Einstein $A$-coefficient of PJB. This profile of the $7.7 \mu \mathrm{m}$-band is furthermore assumed temperature independent. We also added the $16.4 \mu \mathrm{m}-$ AIB detected by ISO towards many sightlines (Moutou et al. 2000; van Kerckhoven et al. 2000). This band is often associated with the "classical" 3 to $13 \mu \mathrm{m}$-AIBs and its intensity is not related to the hot dust continuum appearing 
in more excited objects (see Fig. 1), i.e., as for the other AIBs. We represent the $16.4 \mu \mathrm{m}$-band with a Lorentz profile centered at $609 \mathrm{~cm}^{-1}$, with $F W H M=6 \mathrm{~cm}^{-1}$ (both being observational values, see Moutou et al. 2000) and an Einstein $A$-coefficient of $3.1 \times 10^{-2} \mathrm{~s}^{-1}$ per carbon atom which corresponds to the average value of the laboratory measurements given in Moutou et al. (1996, Table 3). As we will see below, the intensity of the $16.4 \mu \mathrm{m}$-band can constrain the power-law index of the PAH size distribution. At longer wavelengths, we adopted the far-IR $(\lambda \geq 20 \mu \mathrm{m})$ cross-section of Schutte et al. (1993).

The size distribution of PAHs is defined by $\mathrm{d} N=$ $B N_{\mathrm{C}}^{\beta} \mathrm{d} N_{\mathrm{C}}$, i.e., the number of PAHs with a number of C-atoms between $N_{\mathrm{C}}$ and $N_{\mathrm{C}}+\mathrm{d} N_{\mathrm{C}}$. Writing the size distribution with respect to $N_{\mathrm{C}}$ frees us of any assumptions on the PAH geometrical shape ${ }^{1}$. The number of H-atoms per molecule is assumed to be $N_{\mathrm{H}}=f_{\mathrm{H}} \times \sqrt{6 N_{\mathrm{C}}}$ (Omont 1986 , in the case of symmetric PAHs) with $f_{\mathrm{H}}$ the hydrogenation fraction of a molecule: if $f_{\mathrm{H}}=0$ the molecule is completely dehydrogenated, if $f_{\mathrm{H}}=1$ the molecule is fully hydrogenated. Unless otherwise stated, we will assume $f_{\mathrm{H}}=1$. The mass distribution is then straightforwardly found as $\mathrm{d} N \times\left(m_{\mathrm{C}}+m_{\mathrm{H}} \times f_{\mathrm{H}} \sqrt{6 / N_{\mathrm{C}}}\right)$ where $m_{\mathrm{C}}$ is the carbon atomic mass and $m_{\mathrm{H}}$ the atomic mass of hydrogen. The normalization factor, $B$, is determined from the $\mathrm{PAH}$ abundance in terms of the fraction of interstellar carbon $\left([\mathrm{C} / \mathrm{H}]_{\mathrm{ISM}}=2.6 \times 10^{-4}\right.$, Snow \& Witt 1996) locked up in PAHs. Finally, the emergent SED emitted by PAHs is

$S^{\mathrm{e}}\left(\nu^{\prime}\right)=\int_{N_{\min }}^{N_{\max }} S\left(\nu^{\prime}, N_{\mathrm{C}}\right) \mathrm{d} N\left(N_{\mathrm{C}}\right)$

with $N_{\min }$ and $N_{\max }$ the smallest and largest number of Catoms per molecule respectively. We also define the total luminosity emitted by PAHs as $L_{\mathrm{e}}=\int_{0}^{\infty} S_{\nu^{\prime}}^{\mathrm{e}} \mathrm{d} \ln \nu^{\prime}$.

\subsection{The infrared cooling of PAHs}

Upon absorption of a UV-photon, the PAH temperature is raised to a peak value, $T_{\mathrm{p}}$. The molecule then cools rapidly by the emission of IR vibrational mode photons. The peak temperature $T_{\mathrm{p}}$ is found from first principles:

$U_{\mathrm{f}}-U_{\mathrm{i}}=\int_{T_{\mathrm{i}}}^{T_{\mathrm{p}}} C(T) \mathrm{d} T$

where $C(T)$ is the heat capacity of PAHs as given by Dwek et al. (1998, Eq. (A4) of their Appendix), $U_{\mathrm{i}, \mathrm{f}}$ are the initial and final molecular internal energies and $T_{\mathrm{i}}$ the PAH temperature prior to photon absorption or the mean molecular temperature between two photon absorptions. We assumed that $U_{\mathrm{f}}>>U_{\mathrm{i}}$ with $U_{\mathrm{f}}=h \nu$ the energy of

\footnotetext{
${ }^{1}$ Using the grain radius, $a$, as a variable the size distribution reads $\mathrm{d} \mathcal{N} \sim a^{\alpha} \mathrm{d} a$. We thus have the relationship $\beta=(\alpha-$ $d+1) / d$, where $d$ is the dimension of the grain: $d=2$ for planar grains and $d=3$ for spherical grains. For instance, assuming PAHs are planar $\left(a=0.9 \sqrt{N_{\mathrm{C}}}\right)$ a value of -3.5 for $\alpha$ corresponds to $\beta=-2.25$.
}

the absorbed photon. Furthermore, we took $T_{\mathrm{i}}$ to be the PAH temperature at the end of cooling (see the definition below). The heat capacity, as a function of temperature, is approximated by a polynomial in $T$; it is also proportional to $N_{\mathrm{C}}$ but independent of $N_{\mathrm{H}}$, i.e., the contribution of the hydrogen atoms to $C(T)$ is assumed to be always the same whatever the size of the molecule (Dwek et al. 1998). In this case and for a given energy per C-atom in the molecule $\left(h \nu / N_{\mathrm{C}}\right), T_{\mathrm{p}}$ is fixed.

The cooling curve $T_{\mathrm{c}}\left(t, \nu, N_{\mathrm{C}}\right)$ is obtained by numerical integration (vs. temperature) of the energy conservation relationship during cooling,

$P_{\mathrm{e}}\left(T_{\mathrm{c}}\right) \mathrm{d} t=-C\left(T_{\mathrm{c}}\right) \mathrm{d} T$

where $P_{\mathrm{e}}$ is the total power emitted at all wavelengths by the molecule and $\mathrm{d} T$ the temperature drop during the time $\mathrm{d} t$. This simple differential equation determines a family of cooling curves, $T_{\mathrm{c}}$, as a solution, each cooling curve being completely defined by the knowledge of $N_{\mathrm{C}}$ and of the initial condition, i.e., $T=T_{\mathrm{p}}$ at $t=0$.

The power emitted by a $\mathrm{PAH}$ at temperature $T$ is:

$P_{\mathrm{e}}(T)=\int_{0}^{\infty} \sigma_{\nu^{\prime}}^{\mathrm{e}} \times 4 \pi B_{\nu^{\prime}}(T) \mathrm{d} \nu^{\prime}$.

Cooling curves were computed on a grid of 50 points with constant temperature intervals. Under these conditions, we found that the energy conservation requirement:

$\int_{0}^{\infty} P_{\mathrm{e}}\left(T_{\mathrm{c}}(t)\right) \mathrm{d} t=h \nu$

is always met within a few percent.

Each molecule sees its cooling interrupted by the absorption of the next photon which is a stochastic process (Désert et al. 1986). Moreover, gas-grain exchanges must be taken into account (Rouan et al. 1992; Draine \& Lazarian 1998) to reliably estimate the temperature of a $\mathrm{PAH}$ in the low temperature part of the cooling curve. In fact, the emission from the cool (but long-lasting) tail of the cooling curves will peak in the far-IR/submillimeter, far away from the 3 to $20 \mu \mathrm{m}$-range on which we focus here. The detailed treatment of this problem is outside the scope of this paper and we adopt the following simple criterion to truncate our cooling curves. The mean time elapsed between two photon absorptions is $1 / R_{\text {abs }}$ : it is for instance 6.9 and 2.6 hours in the radiation field of NGC 2023 and M17-SW respectively and for a molecule with $N_{\mathrm{C}}=20$. Consequently, all cooling curves have been truncated at $t_{\mathrm{e}}=1 / R_{\mathrm{abs}}$ to account for the repeated absorptions of UV photons by a molecule; $t_{\mathrm{e}}$ decreases as $N_{\mathrm{C}}^{-1}$ because $R_{\text {abs }} \sim N_{\mathrm{C}}$. We thus define the lowest temperature of a $\mathrm{PAH}$ heated by a photon of energy $h \nu$ as $T_{\mathrm{i}}=T_{\mathrm{c}}\left(t_{\mathrm{e}}, \nu, N_{\mathrm{C}}\right)$, i.e., equal to the temperature at the end of the cooling. For instance, in the radiation field of NGC 2023 small PAHs $\left(N_{\mathrm{C}}=20\right)$ eventually reach temperature as low as $15 \mathrm{~K}$ at time $t_{\mathrm{e}}$ whereas bigger PAHs $\left(N_{\mathrm{C}}=500\right)$ remain at around $35 \mathrm{~K}$.

In Fig. 4, we show the cooling curves obtained for various energies per C-atom $E_{\mathrm{C}}=h \nu / N_{\mathrm{C}}$. As expected, 


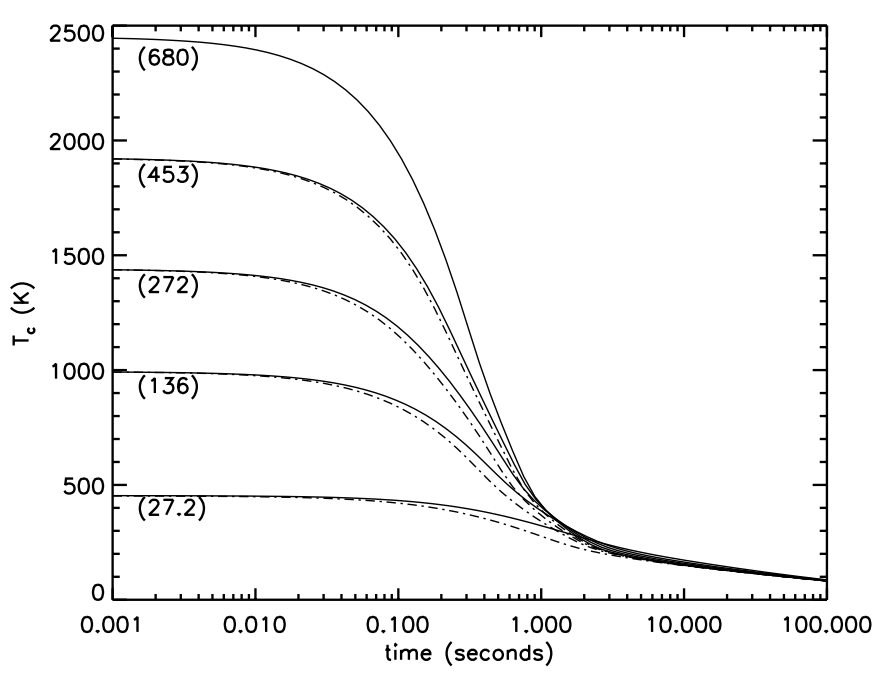

Fig. 4. Cooling curves for various energy per carbon atom (the zero time point has been excluded). The numbers in parenthesis give the value of $E_{\mathrm{C}}=h \nu / N_{\mathrm{C}}$ in $\mathrm{meV} / \mathrm{C}$. The corresponding $\left(h \nu, N_{\mathrm{C}}\right)$ combinations were as follows: a) $E_{\mathrm{C}}=680 \mathrm{meV} / \mathrm{C}$, solid line is $\left(h \nu=13.6 \mathrm{eV}, N_{\mathrm{C}}=20\right)$, b) $453 \mathrm{meV} / \mathrm{C}$, solid $=(13.6,30)$ and dot-dash $=(9.06,20)$, c) $272 \mathrm{meV} / \mathrm{C}$, solid $=(13.6,50)$ and dot-dash $=(5.44,20)$, d) $136 \mathrm{meV} / \mathrm{C}$, solid $=(13.6,100)$ and dot-dash $=(2.72,20)$, e) $27.2 \mathrm{meV} / \mathrm{C}$, solid $=(13.6,500)$ and dot-dash $=(0.54,20)$.

the peak temperatures are identical for a given $E_{\mathrm{C}}$. We note that the $T_{\mathrm{c}}$-curves do not change much for different $N_{\mathrm{C}}$-values while $E_{\mathrm{C}}$ remains fixed: in fact if the emission cross-section were only proportional to $N_{\mathrm{C}}$ all the cooling curves with $\left(h \nu, N_{\mathrm{C}}\right)$ combinations yielding the same $E_{\mathrm{C}}$ would merge. Cooling curves with larger $N_{\mathrm{C}}$ have higher temperatures because emission in the $\mathrm{C}-\mathrm{H}$ modes is less important (in a fully hydrogenated $\mathrm{PAH}$ we have $\left.N_{\mathrm{H}} / N_{\mathrm{C}}=\sqrt{6 / N_{\mathrm{C}}}\right)$. The small variation in the $T_{\mathrm{c}}$-curves at fixed $E_{\mathrm{C}}$ then reflects the decrease of the emission crosssection per carbon atom with increasing size.

\subsection{The temperature distribution of interstellar PAHs}

As noted above, the observed similarity of the AIB profiles in our sample means that the range of $\mathrm{PAH}$ emission temperatures does not vary much: to test this idea, we determine here the temperature distribution of PAHs. Moreover, as we show below, the effect of the size distribution and exciting radiation field parameters are illustrated in a synthetic fashion in the temperature distribution.

The observed AIB spectrum results principally from the superposition of many blackbodies at different temperatures (Eqs. (3) and (5)). Following Eq. (3) it is clear that a given blackbody will have a significant contribution to the emergent spectrum if its temperature $T$ is high and/or if it remains a long time at that temperature: a PAH with a temperature between $T$ and $T+\mathrm{d} T$ (corresponding to the time interval $[t, t+\mathrm{d} t]$ ) will thus contribute to the total emitted luminosity $L_{\mathrm{e}}$ (see the end of Sect. 4.1) with a weight proportional to $P_{\mathrm{e}}(T) \times \mathrm{d} t$ or the fraction of the total available energy, $h \nu$ (the energy of the absorbed photon), that is dissipated between $t$ and $t+\mathrm{d} t$. This definition of the temperature weight is different from previous work (Draine \& Anderson 1985; Désert et al. 1986) which only considered the time that a given grain spends in the temperature interval $[T, T+\mathrm{d} T]$, namely $\mathrm{d} t / \mathrm{d} T$ : the present definition which takes into account the emitted power actually reflects the contribution of a given temperature to the final spectrum.

From Eq. (7), we see that the temperature weight in the total luminosity is simply $C(T) \times \mathrm{d} T$. Taking into account the probability distribution of the exciting photons and the fraction of PAHs containing $N_{\mathrm{C}}$ carbon atoms, we define the temperature weight $w_{\mathrm{T}}$ as:

$w_{T}=\frac{C\left(T_{\mathrm{c}}\right) \delta T}{h \nu} \times \mathcal{P}_{\nu} \delta \nu \times \frac{N_{\mathrm{C}}^{\beta+1} \delta \ln N_{\mathrm{C}}}{N_{\mathrm{PAH}}}$

where $N_{\mathrm{PAH}}$ the total number of PAHs and $\delta T$ is the temperature bin in $T_{\mathrm{c}}, \delta \nu$ the frequency bin of the exciting radiation field and $\delta \ln N_{\mathrm{C}}$ the logarithmic bin of the size distribution. All these bins have been taken as fixed. With this definition we have $\sum_{\left(T, N_{\mathrm{C}}, \nu\right)} w_{T}=1$.

To obtain the PAH temperature distribution in a given exciting radiation field, we build the histogram of all cooling curves for all molecular sizes in temperature bins of constant size $(\Delta T=100 \mathrm{~K})$, each temperature $T$ being given the weight $w_{T}$. The density value in the histogram is then simply $\sum_{T^{\prime}=T}^{T+\Delta T} w_{T^{\prime}}$. The distribution per temperature interval, $p(T)$ is then found by normalizing the histogram so that $\int_{0}^{\infty} p(T) \mathrm{d} T=1$.

From our weight definition, $p(T) \mathrm{d} T$ is the fractional contribution of all PAHs with temperatures in the range $[T, T+\mathrm{d} T]$ to the total emitted luminosity $L_{\mathrm{e}}$.

The PAH temperature distributions, normalized to 1 at their peak values to facilitate the comparison, are shown in Fig. 5: they all present a maximum between 300 and $800 \mathrm{~K}$. The shape of these distributions can be understood as follows. Assuming that all molecules absorb a photon of energy $h \nu$, the temperature weights are proportional to $C(T) \times N_{\mathrm{C}}^{\beta+1}$. At low $T$, all the $C(T)$-curves corresponding to the different $N_{C}$ 's sum up to give $w_{T}$. As the temperature increases, the largest molecules do not contribute anymore to $w_{T}$ because they are too cold: this fade out more than compensates for the rise of $C(T)$ with the temperature and produces a maximum in the temperature distribution.

Figure 5 illustrates the sensitivity of the PAH temperature distribution to the size distribution parameters, $N_{\min }$, $N_{\max }$ and $\beta$ (Figs. 5a to $5 \mathrm{c}$ ) and to the hardness of the exciting radiation field, parameterized as $T_{\text {eff }}$ (Fig. $5 \mathrm{~d}$ ). The associated changes in the AIB spectrum are shown in Fig. 6. In all these figures (except the M17-SW case in Fig. 5d), we used the same dilution factor for the radiation field $\left(W_{\text {dil }}=2.66 \times 10^{-13}\right.$, corresponding to NGC 2023): indeed, as long as one is in the regime of temperature fluctuations, the spectral distribution of the PAH emission spectrum does not depend on the radiation field intensity (Sellgren 1984) while its absolute level scales with it (see Eq. (3)). Moreover, for the purpose of comparison, all the spectra of Fig. 6 have been normalized to 1 at the peak 


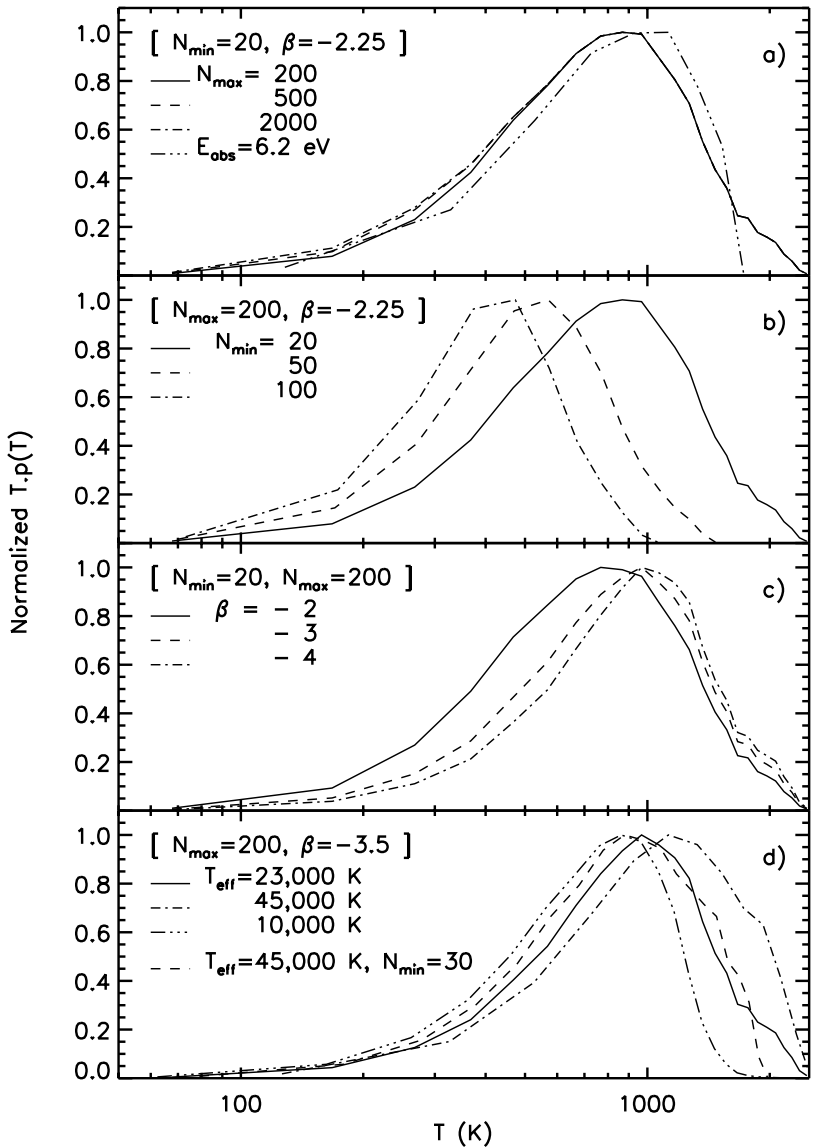

Fig. 5. The distribution of PAH temperatures $T \times p(T)$ normalized to 1 at peak value. In the panels a), b) and c), we used the parameters for the radiation field of NGC 2023 (see Table 1). The fixed parameters of each plot are given in brackets. In a), we vary $N_{\max }$ and the triple-dot dash line shows the effect of assuming that all molecules absorb the same photon $\left(E_{\text {abs }}=6.2 \mathrm{eV}\right)$. Variations of $N_{\text {min }}$ are explored in $\left.\mathbf{b}\right)$. The power-law index, $\beta$, is changed in $\mathbf{c}$ ). In $\mathbf{d}$ ), we explore the effect of $T_{\text {eff }}$ while keeping the dilution factor of NGC 2023 (see text) and $N_{\min }=20$. The solid line corresponds to the case of the NGC 2023 radiation field. The dot-dash line in d) shows the case of the M17-SW radiation field with $N_{\min }=30$, the value required to fit the observed AIB profiles (see Sects. 4.4.1 and 4.4.2).

value of the $7.7 \mu \mathrm{m}$-band because its profile is independent of the PAH temperature (see Sect. 4.1).

In Fig. 5a, we consider the effect of taking the full spectral distribution of exciting photons instead of the same mean absorbed photon energy, $E_{\text {abs }}$ (i.e., $\mathcal{P}_{\nu}=$ $\left.\delta\left[h \nu-E_{\mathrm{abs}}\right]\right)$. In the following, we will refer to the $E_{\mathrm{abs}}{ }^{-}$ approximation whenever it is assumed that each molecule absorbs the same photon of energy $h \nu=E_{\text {abs }}$ at the rate $R_{\text {abs }}$ in the given radiation field. The $E_{\text {abs }}$-approximation only affects the high temperature tail of the distribution. As we will see in Sect. 4.4.2, this tail matters for the width of the $3.3 \mu \mathrm{m}$-band. Nevertheless, the rest of the AIB spectrum is little affected by the $E_{\text {abs-approximation. }}$ $N_{\text {max }}$ has a weak influence on the temperature distribution (Fig. 5b) and its impact, which is most noticeable at long wavelengths ( $\lambda \geq 10 \mu \mathrm{m}$, see Fig. 6a), is easily

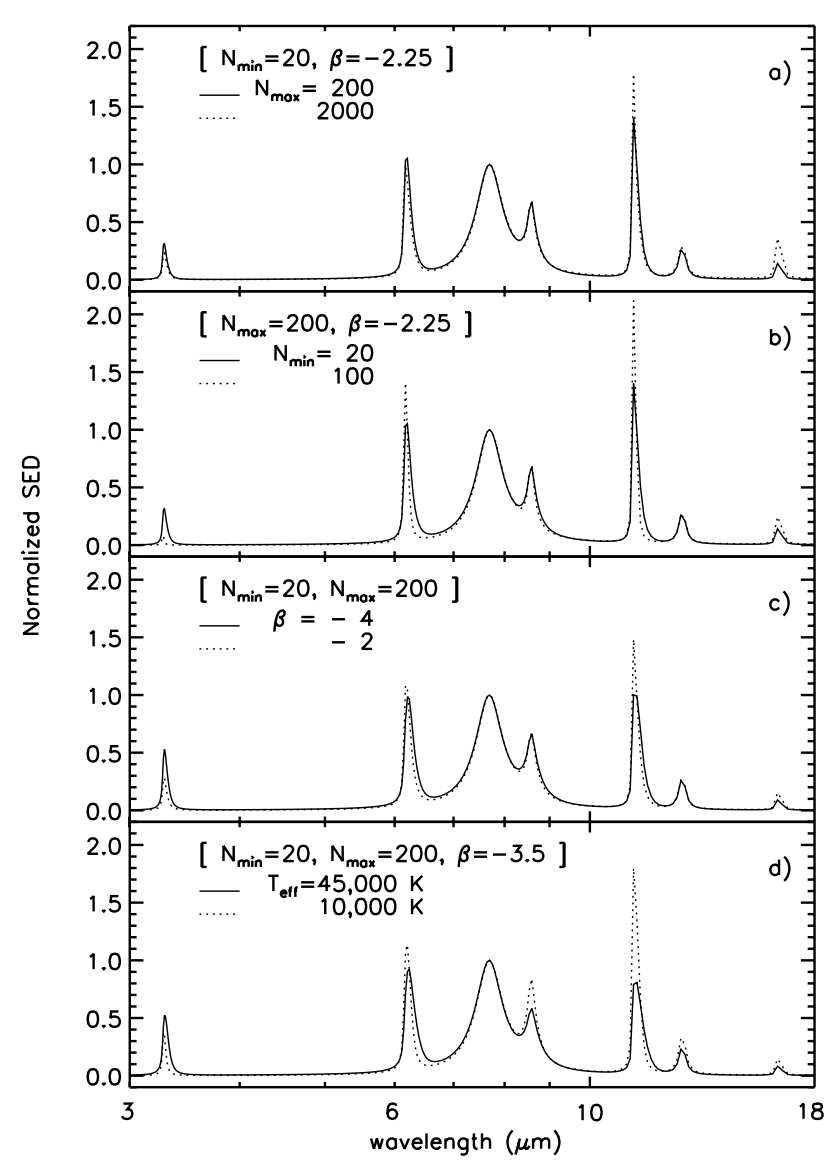

Fig. 6. The spectral energy densities corresponding to the temperature distributions shown in Fig. 5. All the curves have been normalized to 1 at the peak value of the $7.7 \mu \mathrm{m}$-band (see text). In panels a) to c), the exciting radiation field is that of NGC 2023 and we vary the parameters of the PAH size distribution with respect to some reference values, namely, $N_{\min }=20, N_{\max }=200$ and $\beta=-2.25$. In d), we look at the effect of changing the effective temperature of the exciting radiation field while the dilution factor is always equal to that of NGC 2023. In each panel, the parameters kept constant are given in brackets.

drowned by small changes of $\beta$, the index of the powerlaw size distribution of PAHs (see Figs. $5 \mathrm{c}$ and $6 \mathrm{c}$ ). When $\beta$ decreases, the contribution of the smallest PAHs becomes more important and the cold tail of the temperature distribution is somewhat reduced (Fig. $5 \mathrm{c}$ ): this leads to an enhanced $3.3 / 11.3 \mu \mathrm{m}$-band ratio and to somewhat broader bandwidths (Fig. 6c and Figs. 8 to 11). Note that, due to this broadening, the band peak value diminishes (see the $6.2 \mu \mathrm{m}$-band in Fig. 6c) because the band integrated cross-section is conserved with temperature. Both $N_{\text {min }}$ and $T_{\text {eff }}$ (which determines $E_{\text {abs }}$, here $T_{\text {eff }}=10000$ to $45000 \mathrm{~K}$ corresponds to $E_{\mathrm{abs}}=4$ to $9 \mathrm{eV}$ ) affect the hot tail of $p(T)$ (Figs. 5b and $5 \mathrm{~d}$ ) which merely reflects the hottest part of the cooling curve (Fig. 4). This behaviour of $p(T)$ follows from the fact that the PAH peak temperature only depends on the energy content per carbon atom in a molecule, $E_{\mathrm{C}}$ (see Sect. 4.2) and that a typical value for $E_{\mathrm{C}}$ is $E_{\mathrm{abs}} / N_{\min }\left(N_{\min }\right.$ is close to the mean value of $N_{\mathrm{C}}$ because of the steep power-law size 
distribution). In fact, the temperature distribution and the shape of the emission spectrum are similar for different $\left(E_{\mathrm{abs}}, N_{\mathrm{min}}\right)$-couples yielding the same energy per carbon atom. As expected, an increase of $N_{\text {min }}$ leads to colder PAHs (Fig. 5b) which contribute more to the longwavelength bands (Fig. 6b) while an increase of $T_{\text {eff }}$ has the reverse effect (see Figs. 5d and 6d). We pointed out earlier that the PAH temperature distribution must remain unchanged in order to explain the similarity of the observed AIB profiles: we show in Fig. 5 d that this condition is fulfilled if $N_{\min }$ is raised along with $T_{\text {eff }}$. Specifically, an increase of $T_{\text {eff }}$ from $23000 \mathrm{~K}$ (the case of NGC 2023) to $45000 \mathrm{~K}$ (the case of $\mathrm{M} 17-\mathrm{SW}$ ) requires $N_{\min }=20$ to 30 , respectively. In the next sections, we show that this requirement allows to consistently reproduce the overall AIB spectrum as well as the individual band profiles.

\subsection{Modelling the AIB spectrum}

We now present the PAH IR-emission as computed from the formalism described above in the case of NGC 2023 and M17-SW. We selected these two objects because they present the largest contrast in the effective temperature of the exciting radiation field: we therefore expect these spectra to provide the strongest constraints on our model.

\subsubsection{The complete spectra}

Having determined the temperature distribution of a population of PAHs, we can now compute the corresponding IR emission using Eqs. (3) and (5). The PAH size distribution parameters were constrained as follows. We note that the overall shape of the $3-13 \mu \mathrm{m}$ AIB spectrum which is dominated by emission from molecules at $T \geq 400 \mathrm{~K}$ is not much affected by changes in $N_{\max }$ or $\beta$ (see Figs. 6a and 6c). For simplicity, we fix from now on $N_{\max }=200$. First, $N_{\min }$ is fixed so as to reproduce the observed 3.3/11.3 $\mu \mathrm{m}$-band ratio in a given exciting radiation field (described by $T_{\text {eff }}$ ): we require $N_{\text {min }}=20$ in NGC 2023 while $N_{\text {min }}=30$ in M17-SW. The hydrogenation fraction, $f_{\mathrm{H}}$, is constrained from the $7.7 / 11.3 \mu \mathrm{m}$-band ratio: a good match to the observed band ratios is obtained with $f_{\mathrm{H}}=0.8$ for NGC 2023 and $f_{\mathrm{H}}=0.5$ for M17-SW. The index of the power law size distribution, $\beta$, can be constrained from the requirement that the predicted $16.4 \mu \mathrm{m}$-band (on top of the broadband continuum) matches the observed band: we find that $\beta=-3.5$ reproduces the $16.4 \mu \mathrm{m}$-band well in both objects ${ }^{2}$. Unfortunately, individual PAH species show a large spread in the integrated cross-section of the $16.4 \mu \mathrm{m}$-band (see Table 3 of Moutou et al. 1996) which does not constrain $\beta$ : specifically, to match the observed $16.4 \mu \mathrm{m}$-band we require $\beta=-2.25$ for the lowest cross-section value

\footnotetext{
2 This result is not sensitive to the amount of cooling occurring in the far-IR $(\lambda \leq 20 \mu \mathrm{m})$ : multiplying or dividing our far-IR cross-section by 10 did not alter much our match of the $16.4 \mu \mathrm{m}$-band.
}

while $\beta=-6$ for the largest cross-section value. More laboratory work on PAHs is warranted in order to understand the trend of the $16.4 \mu \mathrm{m}$-band profile with respect to $N_{\mathrm{C}}$ and to the various isomer states. As discussed by PJB, an additional constraint on $\beta$ is provided by the intensity and width of the AIB profiles (see the next section). The $\beta$-value can then be adjusted in order to match the peak position and the FWHM of the $3.3 \mu \mathrm{m}$-band (see Sect. 4.4.2): in fact, the value of -3.5 found from the $16.4 \mu \mathrm{m}$-band gives a good fit in the case of NGC 2023 and M17-SW (see Fig. 8).

At this point, it is interesting to note that that the 6 to-13 $\mu \mathrm{m}$ part of the spectrum is produced by molecules with emission temperatures between 400 and $1000 \mathrm{~K}$. During temperature fluctuations, most PAHs go through these temperatures: this is why the maximum of the temperature distribution (Fig. 5) always falls in this range. In fact, the emission of any $\mathrm{PAH}$ with $N_{\mathrm{C}} \leq 160$ shows ratios of band peak values which are quite comparable to the observed 6-13 $\mu \mathrm{m}$ AIB spectrum (see PJB and Draine $\&$ Li 2001). On the other hand, the match of the observed band position and width in the present data sample and in CAM-CVF spectra of similar interstellar regions requires small molecules (see next section). However, taking into account the full size distribution of PAHs will add emission mostly at $3.3 \mu \mathrm{m}$ (from the smaller species, see Fig. 6b) and in the far-infrared (from the larger species, see Fig. 6a) without changing much the $6-13 \mu \mathrm{m}$ region. Thus, in the framework of the present model, the observed invariance of the AIB spectrum in CAM-CVF data $(6-16 \mu \mathrm{m})$ implies that the AIB carriers are small enough to undergo large temperature fluctuations (at least $400 \mathrm{~K}$ ) during which they emit in this wavelength range.

Our best fit SED's are displayed in Fig. 7: we assumed a total column density of $1.8 \times 10^{21} \mathrm{~cm}^{-2}$ (i.e., 1 visible magnitude of extinction) for both NGC 2023 and M17SW with $10 \%$ and $8 \%$, respectively of the interstellar carbon in PAH cations (using $[\mathrm{C} / \mathrm{H}]_{\mathrm{ISM}}=2.6 \times 10^{-4}$, Snow $\&$ Witt 1996). The hydrogenation fraction of PAHs was taken to be $80 \%$ for NGC 2023 and 50\% for M17-SW. To compare in a meaningful fashion the predicted PAH emission to the observed spectrum, the broadband continuum described in Sect. 3 has been added to our model spectrum. This continuum consists of the modified blackbody along with the 1000 and $1450 \mathrm{~cm}^{-1}$ broad profiles (in M17-SW an additional broad profile at $600 \mathrm{~cm}^{-1}$ is required, see Fig. 2). All the main AIBs (at 3.3, 6.2, 7.7, 8.6 and $11.3 \mu \mathrm{m}$ ) are matched within $20 \%$. We note that the model fails to reproduce the $12.7 \mu \mathrm{m}$-band in M17SW: this may be due to an ill-definition of the underlying broadband continuum in this complex spectral region (see Fig. 2). The AIB spectrum corresponding to the neutral PAHs (we took the IR cross-sections from Léger et al. 1989b) is shown in the case of NGC 2023 with a PAH abundance corresponding to $6 \%$ of the interstellar carbon, with $N_{\min }=20, N_{\max }=200$ and $\beta=-3.5$. The $N_{\text {min-value was fixed so as to reproduce the position and }}$ width of the $3.3 \mu \mathrm{m}$-band (see next section) and it is the 


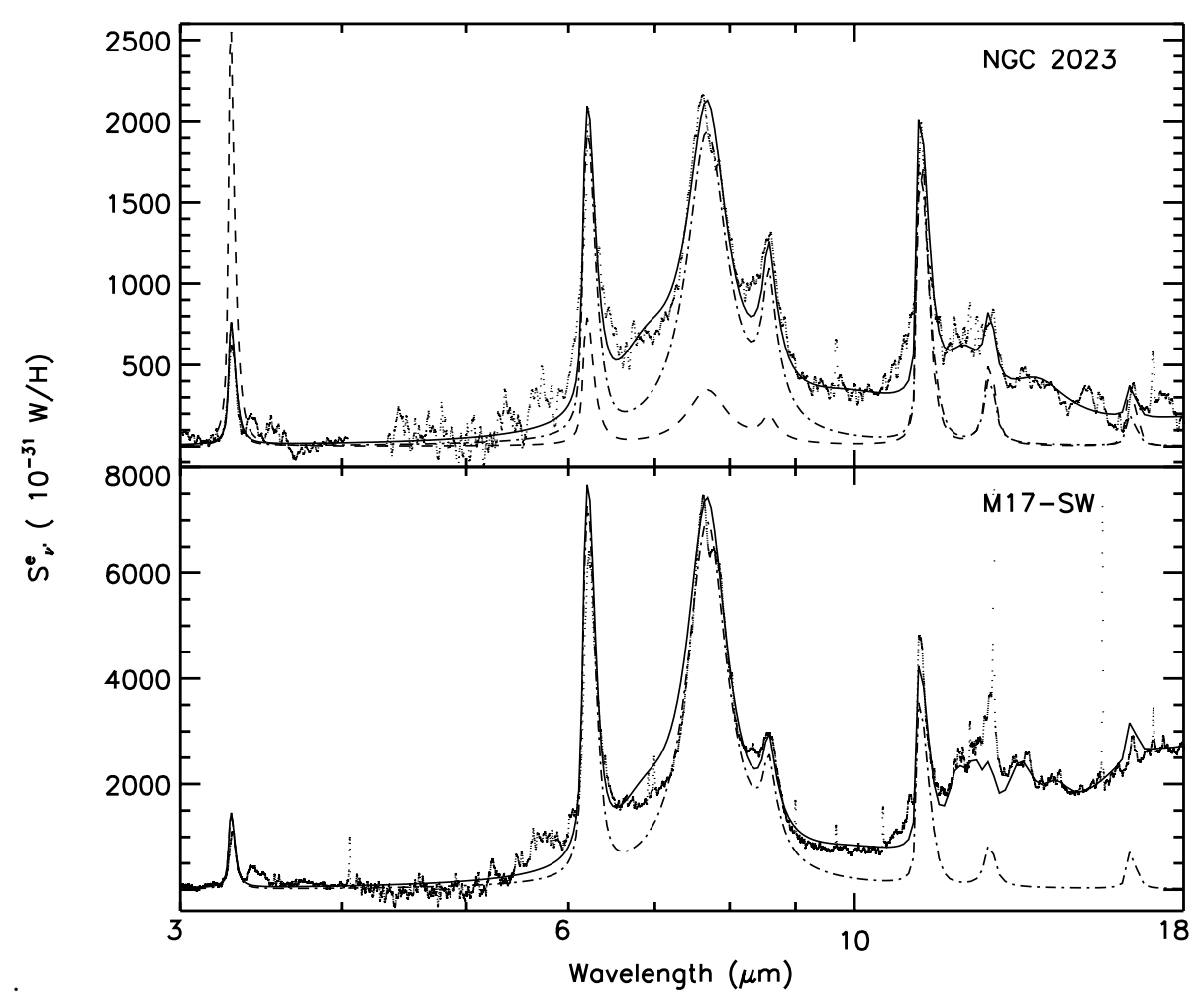

Fig. 7. The spectral energy density in Watt per hydrogen for our sample of objects from the SWS data (dotted curve) and assuming a column density of $1.8 \times 10^{21} \mathrm{~cm}^{-2}$. The resolving power $(\lambda / \Delta \lambda)$ is 200 for NGC 2023 and 500 for M17-SW. The model PAH emission is the dot-dash line. The solid line shows the model complete spectrum which includes the broadband continuum described in Sect. 3. We took $N_{\max }=200$ and $\beta=-3.5$ for both objects. For NGC 2023, we take $N_{\min }=20$, $f_{\mathrm{H}}=0.8$ and $10 \%$ of the interstellar carbon in PAHs while we require $N_{\min }=30, f_{\mathrm{H}}=0.5$ and $8 \%$ of the interstellar carbon in PAHs for M17-SW (see text). In the upper panel, the dashed line shows the case of neutral PAHs from our model with $N_{\min }=20, N_{\max }=200, \beta=-3.5, f_{\mathrm{H}}=1$. For clarity, the SED of neutral PAHs has been normalized to the peak value of the $11.3 \mu \mathrm{m}$-band in the SED of ionized PAHs (dot-dash line). This normalization amounts to a PAH abundance representing $6 \%$ of the interstellar carbon.

same as for the cations. In the case of neutral PAHs, the $\mathrm{C}-\mathrm{C} / \mathrm{C}-\mathrm{H}$ band ratio is reduced by about one order of magnitude with respect to the cations. Matching the observed AIB with neutral PAHs would require a very low hydrogen coverage $\left(f_{\mathrm{H}} \leq 0.1\right)$ which would produce a strongly discrepant $8.6 \mu \mathrm{m}$-band and also would be at odds with theoretical predictions on the hydrogen coverage of PAHs (Allain et al. 1996).

In the following, we compare in detail the profiles of the observed $3.3,6.2,8.6$ and $11.3 \mu \mathrm{m}$-AIBs to our model results: we restrict our comparison to these bands because they are well defined in the data and involve a small number of Lorentz fit components.

\subsubsection{Individual AIB profiles}

To do a detailed model-to-data comparison, we now study the individual AIB profiles. Due to anharmonic couplings, the line profiles of PAH vibrational transitions are redshifted and broadened as the temperature of the molecule increases and both follow a linear law (Joblin et al. 1995). The temperature dependent broadening laws of PJB used in this modelling do not include the rotational width. The centroid and width of each band profile are given by $\nu(T)=\nu_{0}+\chi_{\mathrm{c}} \times T$ and $\Delta \nu(T)=\Delta \nu_{0}+\chi_{w} \times T$ respectively. The coefficients of the linear $T$-laws have been measured in the laboratory on a restricted sample of small $\left(N_{\mathrm{C}} \leq 32\right)$, neutral PAHs: we assume that the temperature dependence of the vibrational band profile are the same for all PAH sizes as well as for cations and neutrals. To match the position of the observed AIB profiles, we had to slightly change the band centers at $0 \mathrm{~K}$ : namely, for the $3.3,6.2,8.6$ and $11.3 \mu \mathrm{m}$-bands we took $\nu_{0}=3079$ (3076), 1636 (1627), 1169 (1141) and 899 (896) $\mathrm{cm}^{-1}$ where the laboratory values are in parenthesis. Such shifts are reasonable in view of the laboratory results on the spread in band positions for different species.

In order to highlight the match in band width and position, our model profiles are compared to the observed AIBs in a normalized fashion. For all the results of best fit models represented, the peak absolute intensities do not deviate by more than $20 \%$ from the observations (Fig. 7). We use the $N_{\text {min-values derived from the complete spec- }}$ tra, i.e., $N_{\min }=20$ and 30 for NGC 2023 and M17-SW respectively. Noting that $N_{\min }$ has a strong effect on the PAH emission (Figs. 5c and 6c and Figs. 8c to 11c), it is remarkable that the same value allows to match both the absolute peak intensity and the profile shape of the $3.3 \mu \mathrm{m}$-band. The value of $\beta$ is then chosen so as to 


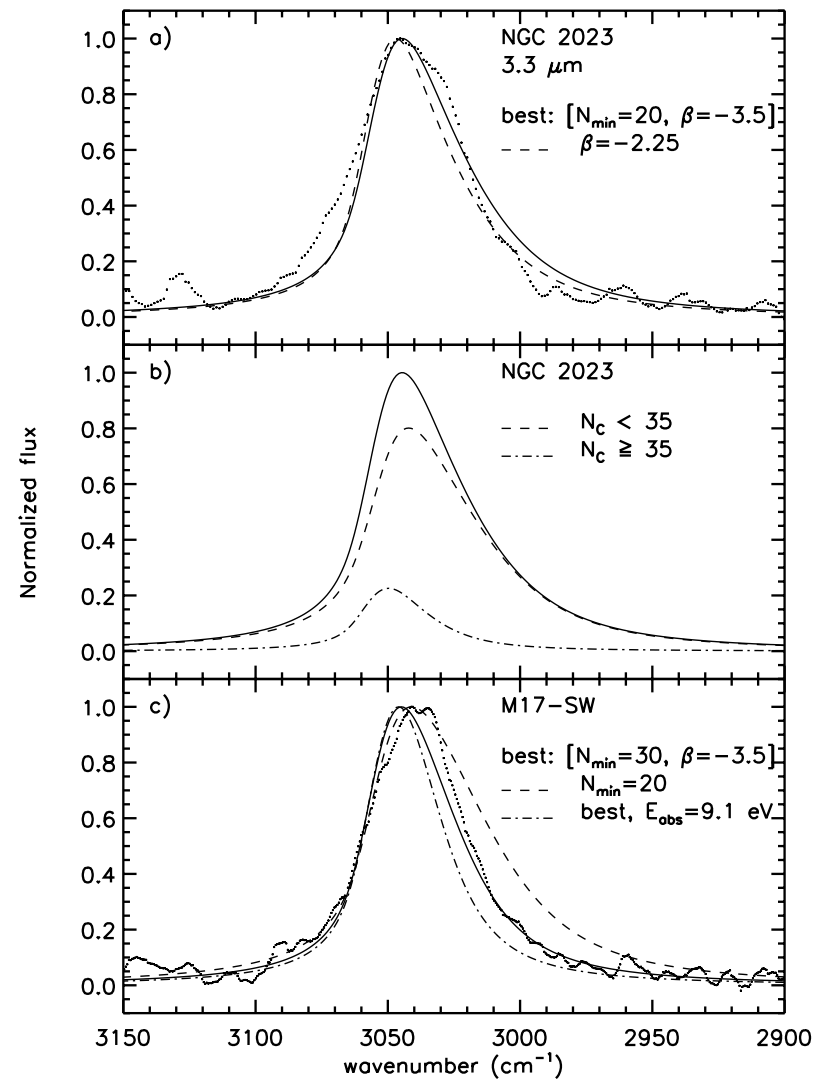

Fig. 8. The normalized profile of the $3.3 \mu \mathrm{m}$-AIB as seen by ISO-SWS compared to our model emission profile also normalized to one (see text). The contribution of the continuum and of all bands other than the $3.3 \mu \mathrm{m}$ have been subtracted. The resolving power of the data $(\lambda / \Delta \lambda)$ is 200 for NGC 2023 and 500 for M17-SW. For all the model profiles, we took $N_{\max }=200$. In a), the SWS spectrum is the dotted curve, the result of our model best fit with $N_{\min }=20$ and $\beta=-3.5$ is the solid line and the dashed line represents the case where $\beta=-2.25$ (while $N_{\min }=20$ ). In $\mathbf{b}$ ), we show the contribution of the small $\left(20 \leq N_{\mathrm{C}} \leq 35\right.$, dashed line) and large (35 $\leq N_{\mathrm{C}} \leq 200$, dot-dashed line) PAHs to the model best fit of NGC 2023. In c), the observed $3.3 \mu \mathrm{m}$-band profile of M17SW (dotted curve) is shown as well as our model best fit (solid line) with $N_{\min }=30$ and $\beta=-3.5$. The model profile for $N_{\min }=20$ (while $\beta=-3.5$ ) is shown as the dashed line. The dot-dashed line shows a model using the $E_{\text {abs-approximation }}$ $\left(E_{\text {abs }}=9.1 \mathrm{eV}\right.$, see Sect. 4.3$)$ with $N_{\min }=30$ and $\beta=-3.5$.

obtain a good match of the width of all bands: we find that $\beta=-3.5$ is a good compromise. It must be noted, however, that the values of $N_{\min }$ and $\beta$ are strongly dependent on the adopted profile temperature laws.

Figures 8 to 11 show our model representation of the AIB profiles. The contribution of vibrational hot bands are not included in our model profiles: in the case of the 6.2 and $11.3 \mu \mathrm{m}$-bands, hot bands provide an additional broadening of less than $5 \mathrm{~cm}^{-1}$ (PJB). In Fig. 8c, we note the effect of making the $E_{\text {abs-approximation (i.e., all }}$ molecules are heated by photons of the same energy $E_{\text {abs }}$, see Sect. 4.3): the lack of the hot tail in the PAH temperature distribution yields a narrower band profile. Hence, using this approximation would lead to an underestimate of $N_{\min }$ : e.g., in the case of NGC $2023, N_{\min }=15$ is required

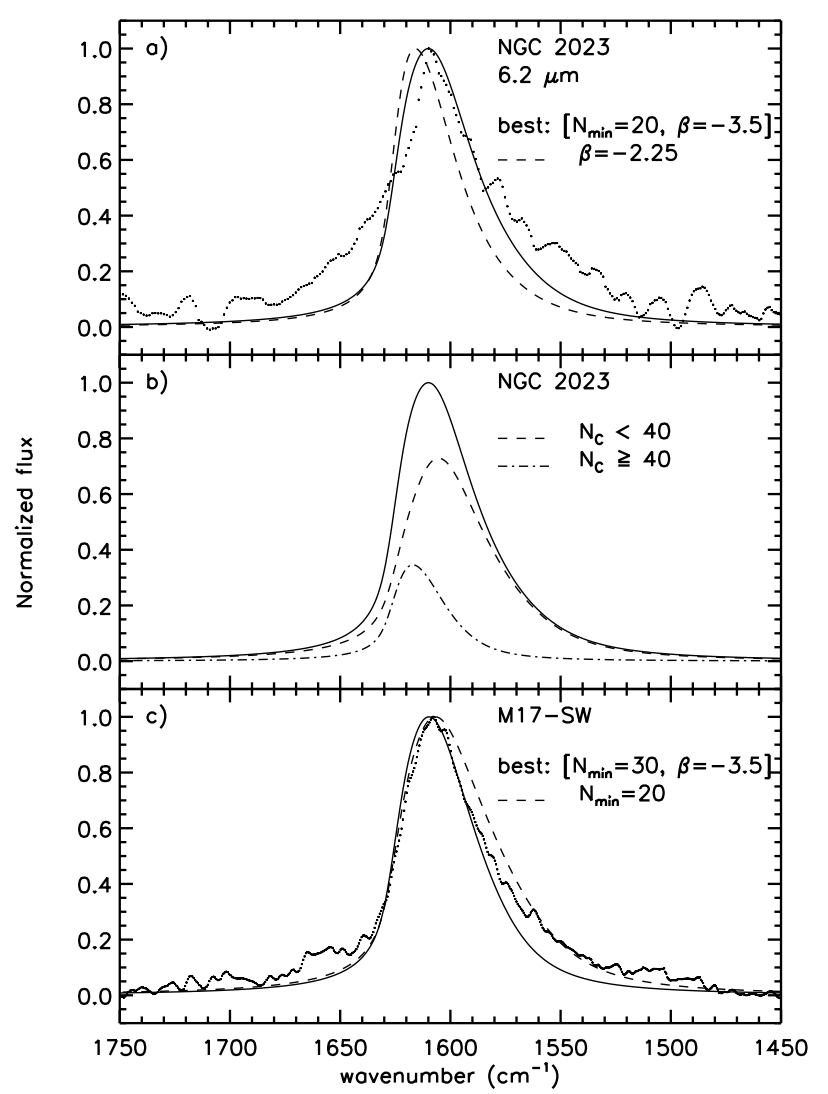

Fig. 9. Same as Fig. 8 for the $6.2 \mu \mathrm{m}$-band. In the case of M17-SW, the profile for the $E_{\text {abs-approximation has not been }}$ represented.

to match the observed $3.3 \mu \mathrm{m}$-band if one makes the $E_{\text {abs }}{ }^{-}$ approximation. This approximation has, however, negligible consequences for the other bands. The effect of a larger $\beta,-2.25$ (corresponding to the classical power-law exponent of -3.5 when the size distribution is expressed in terms of the grain radius, Mathis et al. 1977) is shown as the dashed line in Figs. 8a to 11a: a low $\beta$-value is clearly required to match the observed band profiles. Decreasing $\beta$, however, has a moderate impact on the band profiles because the then enhanced contribution of small molecules is compensated by a strong increase of the band width leading to a decrease in intensity (the integrated cross-sections of the IR bands, $\sigma \times \Delta \nu$, are assumed to be conserved with temperature).

The harder radiation field of M17-SW results in PAHs at higher temperatures (see Fig. 5d), consequently the model AIB-profiles are clearly too broad and redshifted (see the dashed line in the bottom panel of Figs. 8 to 11) if we use the same size distribution as for NGC 2023. For the profile width and position, $N_{\min }=30$ is also required in order to match the observed AIBs of M17-SW. This change of $N_{\min }$ has a physical interpretation: the photodissociation and fragmentation rates rise steeply with the molecular temperature (Léger et al. 1989a). The $N_{\min }$ values we find are smaller than the theoretical predictions of Allain et al. (1996).

Except for the $8.6 \mu \mathrm{m}$-band, all AIB profiles are well explained by the PAH emission model. The hot (small) 


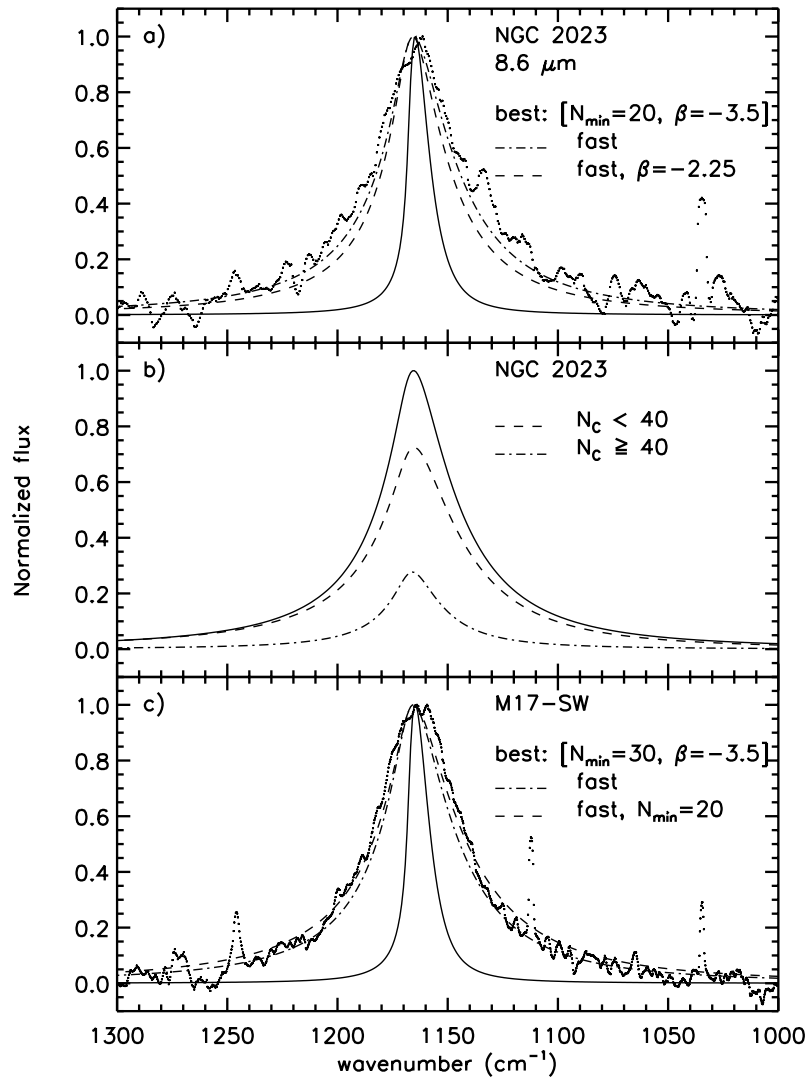

Fig. 10. Model results for the $8.6 \mu \mathrm{m}$-band. The data is displayed as in Fig. 8 with the following changes. In a), the solid line shows the model profile if the bandwidth temperature dependence derived from laboratory results is used. The dotdash line shows the model profile for a hypothetical broadening 5.5 times faster (see text) and the dashed line corresponds to $\beta=-2.25$ case for the fast broadening law. In $\mathbf{b}$ ), we show the contribution of small and large PAHs in the case of the fast broadening law. Finally, the display in c) is the same as in $a$ for the M17-SW case except for the dash line where $N_{\min }=20$ and $\beta=-3.5$ with the fast broadening law. The unresolved lines at 1035,1110 and $1245 \mathrm{~cm}^{-1}$ correspond to $0-0 \mathrm{~S}(3)$ of $\mathrm{H}_{2}$, a forbidden line of [ArIII] and $0-0 \mathrm{~S}(2)$ of $\mathrm{H}_{2}$.

molecules dominate the emission profiles, in particular in the AIB wings; they also produce the observed red asymmetry (6.2 and $11.3 \mu \mathrm{m}$-bands). The cool (large) molecules contribute the blue wing of the AIB. We want to stress that this match of the AIBs is obtained by using the crosssections and profile temperature dependence measured on small molecules: no additional broadening, reflecting the decrease of the level lifetime with increasing molecular size (see Sect. 5), has been taken into account here.

The observed width of the $8.6 \mu \mathrm{m}$-band is not at all reproduced by the temperature broadening law derived from laboratory work. In fact, a broadening 5.5 times faster $\left(\chi_{w}=6.0 \times 10^{-2} \mathrm{~cm}^{-1} / \mathrm{K}\right)$ with temperature would match the observations (see Fig. 10). In this latter case, we note that (i) the symmetrical shape of the $8.6 \mu \mathrm{m}$-band is well explained by the slow redshift of the band centroid with temperature $\left(\chi_{\mathrm{c}}=0.84 \times 10^{-2} \mathrm{~cm}^{-1} / \mathrm{K}\right.$ whereas the other AIBs have 2 to $3 \times 10^{-2} \mathrm{~cm}^{-1} / \mathrm{K}$ ) and (ii) the emission

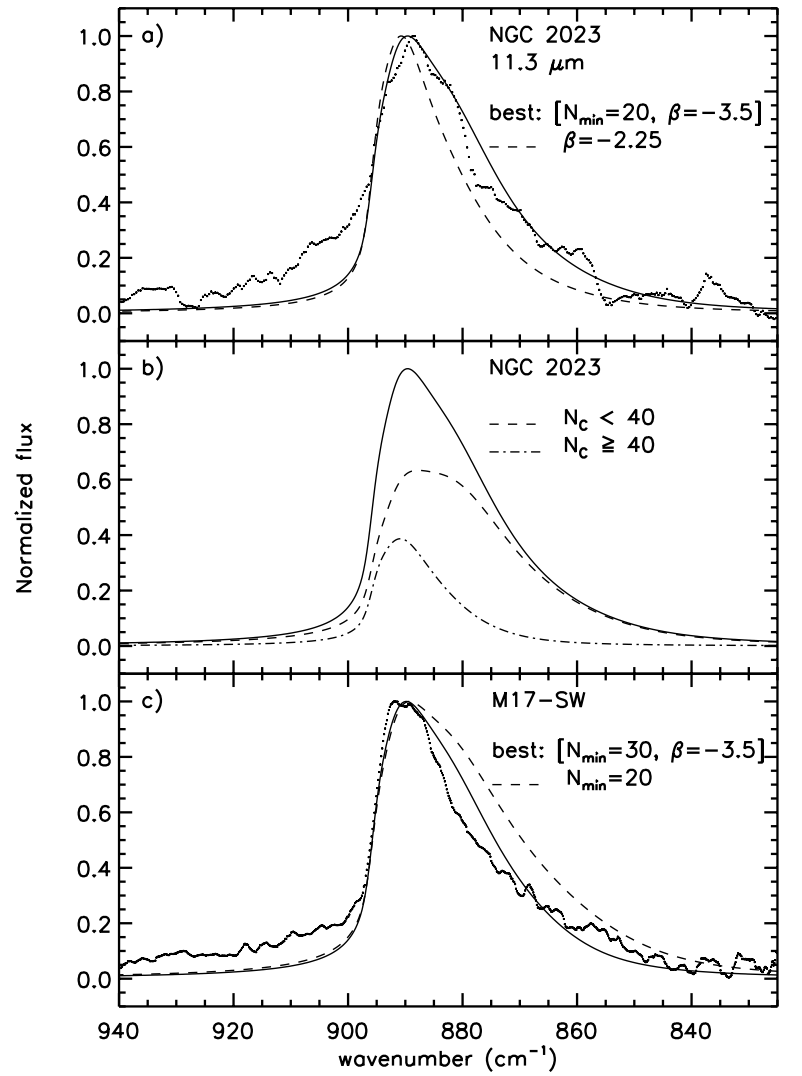

Fig. 11. Same as Fig. 9 for the $11.3 \mu \mathrm{m}$-band. Note that the $11 \mu \mathrm{m}$-AIB has not been withdrawn from the data.

of large molecules dominates the core of the $8.6 \mu \mathrm{m}$-band (conversely to the other AIBs).

We have not modelled the $7.7 \mu \mathrm{m}$-band because it includes several components whose assignment to features in laboratory spectra of PAHs is not straightforward. However, it is interesting to note that the observed width (23 to $29 \mathrm{~cm}^{-1}$ ) of the $7.6 \mu \mathrm{m}$-band (see Sect. 3 ) is comparable to the width predicted by PJB.

We emphasize here that the model profile of a PAH emission band is the result of the superposition of many Lorentz profiles conversely to the implicit assumption behind the decomposition performed in Sect. 3. Consider for instance a PAH containing $N_{\mathrm{C}}$ carbon atoms which has been heated by a photon of energy $h \nu$ : the emerging band profile of this molecule will be the sum of all the Lorentz profiles corresponding to the temperatures of the cooling curve, $T_{\mathrm{c}}\left(t, \nu, N_{\mathrm{C}}\right)$ (i.e., the time integral in Eq. (3)). The total band profile of a PAH interstellar population is eventually obtained from the weighted sum of the emerging band profiles from all molecules, for all energies of the exciting photon (see Eqs. (3) and (5)).

\section{Discussion and summary}

We have decomposed the ISO-SWS spectra of a number of objects covering a wide range of excitation conditions in order to study the individual profiles of the Aromatic Infrared Bands (AIBs) between 3 and $13 \mu \mathrm{m}$. All spectra have been decomposed coherently into Lorentz profiles and a broadband continuum. We find that the individual 
profiles of the main AIBs at 3.3, 6.2, 8.6 and $11.3 \mu \mathrm{m}$ are well represented with at most two Lorentzians. The $7.7 \mu \mathrm{m}$-AIB has a more complex shape and requires at least three Lorentz profiles. We find that the AIB positions and widths are stable to within a few $\mathrm{cm}^{-1}$ (see Table 2) over a range of radiation field hardness $\left(T_{\text {eff }}=23000\right.$ to $45000 \mathrm{~K}$ ) thus confirming results gathered with data at lower spectral resolution (Boulanger et al. 1998a; Uchida et al. 2000; Chan et al. 2000). This spectral decomposition with a small number of Lorentz profiles implicitly assumes that, (i) the AIBs arise from a few vibrational bands common to many carriers and, (ii) that most of the bandwidth arises from a single carrier. Boulanger et al. (1998b) recently proposed that the AIBs are the intrinsic profiles of resonances in small carbon clusters containing more than 50 C-atoms. This interpretation can be tested by comparing the AIB profile parameters (band position and width) given in this work to laboratory data on relevant species when it becomes available.

This spectral decomposition, which separates the AIB emission from the underlying continuum, allowed us to do a detailed comparison of the observed AIB spectrum with the predictions of the PAH model where the AIB carriers are free-flying aromatic molecules emitting during temperature fluctuations. This model uses recent laboratory data and assumes that PAHs are predominantly in cationic form as is expected from theoretical work (Bakes \& Tielens 1994; Dartois \& d'Hendecourt 1997). Within this framework, the position and width of the AIBs are rather explained by a redshift and a broadening of the $\mathrm{PAH}$ vibrational bands as the temperature of the molecule increases (Joblin et al. 1995). The observed similarity in the AIB profiles thus requires that some process renders the temperature distribution of PAHs rather constant in the interstellar regions considered here. We first derived the temperature distribution for a population of interstellar PAHs. In particular, we show that the hot tail of the temperature distribution of PAHs (which determines the AIB spectrum) depends sensitively on $N_{\min }$ and $T_{\text {eff }}$ which are respectively the size of the smallest PAH (in terms of the number of C-atoms in the molecule) and the effective temperature of the exciting radiation field. The size of the largest PAH, $N_{\max }$ (by number of C-atoms), and the index of the power law size distribution (expressed in terms of the number of $\mathrm{C}$-atoms per molecule), $\beta$, were found to have little impact on the overall AIB emission.

We compared our model results to the data in the two extreme cases of our sample: NGC $2023\left(T_{\text {eff }}=23000 \mathrm{~K}\right)$ and $\mathrm{M} 17-\mathrm{SW}\left(T_{\text {eff }}=45000 \mathrm{~K}\right)$. We are able to reproduce the spectral distribution of the AIB emission with $N_{\text {min }}=20$ in NGC 2023 and $N_{\min }=30$ in M17-SW and a PAH abundance amounting to 10 and $8 \%$ of the interstellar carbon (these latter values are in good agreement with the constraints set by the extinction curve, Joblin et al. 1992; Verstraete et al. 1992; and the infrared emission, Désert et al. 1990. An abundance twice as high is required in the case of the cold interstellar medium, Dwek et al. 1997). The minimum PAH size $N_{\min }$ was found from the requirement that the observed 3.3/11.3 $\mu \mathrm{m}$-band be well matched. This change in $N_{\text {min }}$ may reflect the enhanced photodestruction rate of small PAHs in regions with harder radiation fields (small PAHs reach higher temperatures and evaporate efficiently). Using the same $N_{\text {min }^{-}}$ values, we find that all AIB profile shapes, except for the $8.6 \mu \mathrm{m}$-band, can be explained with the temperature dependence of the band position and width measured in the laboratory. We also show that the PAH size distribution must be very steep $(\beta=-3.5)$ in order to account for the observed bandwidths: in our model representation, the AIB profiles are thus mostly contributed to by small PAH species. The case of the $8.6 \mu \mathrm{m}$-band is anomalous: first, the laboratory cross-section is 3 times too weak to explain the observed AIB ratios; in addition, we find that the broadening of this band with the temperature should be 5.5 times faster in order to match the profile width seen in the SWS data. More laboratory work is required to explain this issue.

In summary, using the present best knowledge of $\mathrm{PAH}$ spectroscopy, it is possible to account for the AIB spectrum towards bright interstellar objects where the fraction of singly-ionized PAHs is high. We emphasize that both the spectral shape of the AIB spectrum as well as the individual AIB shapes are explained consistently with a single set of parameter $\left(N_{\min }\right.$ and $\left.\beta\right)$ values. In this context, the remarkable stability of the AIB profiles arises if the hot tail of the $\mathrm{PAH}$ temperature distribution remains essentially the same, whatever the exciting radiation field (the low-temperature side corresponding to the larger molecules is practically unaffected by changes in the radiation field). This requirement is naturally met while considering the photodestruction of PAHs: when the energy per bond (which is directly proportional to the molecular temperature) is sufficient the molecule efficiently loses its atoms. A consequence of this is that interstellar PAHs would predominantly be destroyed by thermal evaporation (photo-thermo-dissociation, see Léger et al. 1989a) rather than non-equilibrium processes like direct photodissociation (Buch 1989) or Coulomb explosion (Leach 1989).

These results must however be placed in the broader context of ISO observations which include less irradiated regions $(\chi=1$ to 1000). Indeed, ISOCAM-CVF and ISOPHOT-S spectra of faint AIB emission highlight the pre-requisites of this work. Namely, two strong assumptions have been made in the present model:

1. the behaviour of the band profiles with the temperature (measured on small molecules only, $N_{\mathrm{C}} \leq 50$ ) is the same for all PAH sizes. This assumption applies for all the AIBs except the $3.3 \mu \mathrm{m}$ : in that case the band profile is actually dominated by the contribution of small species (see Fig. 8b);

2. all PAHs have the infrared emissivity of singly ionized cations.

Assumption 1 is questionable because the intrinsic bandwidth $\left(\Delta \nu_{0}\right.$, see Sect. 4.4.2) depends on the density of states and on the rate of internal conversion processes 
(e.g., Léger et al. 1989a). In particular, at a given internal energy of the molecule, the density of vibrational states is known to increase steeply for larger and larger molecules (e.g., Cook \& Saykally 1998). Many isoenergetic molecular levels may then couple together and eventually lead to a decrease of the level lifetime (Smalley 1983). Hence, relaxing the first assumption will probably result in a significant broadening of the model band profiles; this will require a larger minimum $\mathrm{PAH}$ size in order to reproduce the observed width of AIBs. Increasing $N_{\text {min }}$, the $3.3 \mu \mathrm{m}$ band emission will decrease rapidly because larger (colder) molecules emit more at longer wavelengths and we will then fail to match the intensity of the observed $3.3 \mu \mathrm{m}$ band. This problem will not be alleviated by assuming a $\mathrm{PAH}$ ionized fraction of less than 1, (i.e., not all PAHs are cations): neutral PAHs, which have strong $\mathrm{C}-\mathrm{H} / \mathrm{C}-\mathrm{C}$ band ratios, (typically 10 times larger than in cations, Langhoff 1996; Pauzat et al. 1997; Hudgins \& Sandford 1998), will not reproduce the observed $11.3 / 7.7 \mu \mathrm{m}$-band ratio. This relates to the second assumption of the present model.

We have assumed that PAHs are all singly ionized (cations) throughout our data sample. Such a choice is required to reproduce the observed band ratios, in particular the $\mathrm{C}-\mathrm{H} / \mathrm{C}-\mathrm{C}$ ratio (Langhoff 1996; Allamandola et al. 1999). Yet, this requirement is not fully consistent with theoretical predictions (Dartois et al. 1997) of the $\mathrm{PAH}$ ionized fraction along our lines of sight, namely, 0.5 (50\% of PAHs are cations). Furthermore, this problem becomes even more acute in the context of other observations spanning a broader range of physical conditions: ISOCAM-CVF and ISOPHOT-S data (Boulanger et al. 1998a, 1999; Chan et al. 2000; Miville-Deschênes et al. 1999; Onaka et al. 1999, 2000, Onaka 2000; Uchida et al. 1998, 2000) obtained towards regions with $\chi=1$ to $10^{5}$ (from the diffuse interstellar medium to $\mathrm{H}$ II region interfaces and reflection nebulae) show that the $\mathrm{C}-\mathrm{H} / \mathrm{C}-\mathrm{C}$ ratio of the AIBs is roughly constant and always corresponds to that of $\mathrm{PAH}$ cations. Over such a large range of UV radiation flux, the state of PAHs is actually expected to change from fully neutral to fully ionized. The ionized fraction of PAHs is determined by the value of $\gamma=\chi \sqrt{T} / n_{\mathrm{e}}$ with $T$ the gas temperature and $n_{\mathrm{e}}$ the electron density (this parameter is proportional to the ratio of the ionization rate to the recombination rate of PAHs, Bakes \& Tielens 1994). To keep the AIB band ratios constant, and hence the ionized fraction of PAHs, requires that the ratio $\sqrt{T} / n_{\mathrm{e}}$ can vary over five orders of magnitude in order to compensate the variation of $\chi$. In reality, $T$ probably does not change by more than 2 orders of magnitudes: then $n_{\mathrm{e}}$, which reflects the density variations mostly, would have to vary by three orders of magnitude across the regions observed to keep $\gamma$ constant. Such large density contrasts at large scale are at odds with what is currently known of the structure of these interstellar regions.

More generally, the predicted strong variability of the physico-chemical state (ionization, dehydrogenation) of $\mathrm{PAHs}$ in space is not reflected in the recent ISO spec- troscopy database which covers a variety of astrophysical conditions. This prediction was based on studies of small PAHs $\left(N_{\mathrm{C}} \leq 50\right)$, the only species currently accessible for investigation either in the laboratory or with quantum chemistry. As shown by Schutte et al. (1993), the 6 to $16 \mu \mathrm{m}$-AIB spectrum is also contributed to by large PAHs $\left(N_{\mathrm{C}} \geq 100\right)$ while the $3.3 \mu \mathrm{m}$-AIB is dominated by the smallest species $\left(N_{\mathrm{C}} \leq 35\right.$, see Fig. 8$)$ : this other solution (large "PAHs" or carbon clusters) to the 6 to $16 \mu \mathrm{m}$-AIB spectrum may alleviate the present difficulties. However, the spectroscopical and structural properties of carbon clusters are poorly known at present: the ISO spectroscopic database is ideally suited to identify plausible candidates and stimulate future work on the physics and chemistry of carbon clusters.

Acknowledgements. We are grateful to François Boulanger, Alain Abergel, Christine Joblin and Anthony Jones for many stimulating discussions and our referee for helpful comments. We also thank the MPE-SDC (Garching) and the DIDAC (Groningen) for their constant support in the data reduction phase and with the use of SWS-IA3. SWS-IA3 is a joint developement of the SWS consortium. Contributing institutes are SRON, MPE, KUL and the ESA Astrophysics division. K. S. gratefully acknowledges support from a NATO collaborative Research Grant nr. 951347.

\section{References}

Allain, T., Leach, S., \& Sedlmayr, E. 1996, A\&A, 305, 616

Allamandola, L. J., Tielens, A. G. G. M., \& Barker, J. R. 1985, ApJ, 290, L25

Allamandola, L. J., Hudgins, D. M., \& Sandford, S. A. 1999, ApJ, 511, L115

Bakes, E. L. O., \& Tielens, A. G. G. M. 1994, ApJ, 427, 822

Beintema, D. A., van den Ancker, M. E., Molster, F. J., et al. 1996, A\&A, 315, L369

Bernard, J. P., d'Hendecourt, L. B., \& Léger, A. 1989, A\&A, 220,245

Bernard, J. P., Boulanger, F., \& Puget, J. L. 1993, A\&A, 277, 609

Bohren, C. R., \& Huffman, D. R. 1983, Absorption and scattering of light by small particles, 228 (Wiley \& Sons)

Boulanger, F., Reach, W. T., Abergel, A., et al. 1996, A\&A, 315, L325

Boulanger, F. 1998a, in Star Formation with ISO, ed. J. L. Yun, \& R. Liseau, ASP Conf. Ser., 132, 15

Boulanger, F., Boissel, P., Cesarsky, D., \& Ryter, C. 1998b, A\&A, 339, 194

Boulanger, F., Abergel, A., Cesarsky, D., et al. 1999, in ISO beyond point sources: Studies of extended IR emission, ed. R. J. Laureijs, K. Leech, \& M. F. Kessler, ESA SP-455

Buch, V. 1989, ApJ, 343, 208

Buss, R. H., Allen, M., McCandliss, S., et al. 1994, ApJ, 430, 630

Cesarsky, C. J., Abergel, A., Agnèse, P., et al. 1996, A\&A, 315, L32

Cesarsky, D., Lequeux, J., Abergel, A., et al. 1996a, A\&A, 315, L305

Cesarsky, D., Lequeux, J., Abergel, A., et al. 1996b, A\&A, 315, L309 
Cesarsky, D., Lequeux, J., Ryter, C., \& Gérin, M. 2000a, A\&A, $354, \mathrm{~L} 87$

Cesarsky, D., Jones, A. P., Lequeux, J., \& Verstraete, L. 2000b, A\&A, 358, 708

Chan, K.-W., Roellig, T. L., Onaka, T., et al. 2000, in ISO beyond the peaks: the 2nd ISO workshop on analytical spectroscopy, ESA SP-456 and [astro-ph/0008335]

Cook, D. J., \& Saykally, R. J. 1998, ApJ, 493, 793

Crété, E., Giard, M., Joblin C., et al. 1999, A\&A, 352, 277

Dartois, E., \& d'Hendecourt, L. 1997, A\&A, 323, 534

De Frees, D.J., Miller, M.D., Talbi, D., Pauzat, F., \& Ellinger, Y. 1993, ApJ, 408, 530

Désert, F. X., Boulanger, F., \& Shore, S. N. 1986, A\&A, 160, 295

Draine, B. T., \& Anderson, N. 1985, ApJ, 292, 494

Draine, B. T., \& Lazarian, A. 1998, ApJ, 508, 157

Draine, B. T., \& Li, A. 2001 [astro-ph/0011318]

Dwek, E. Arendt, R. G., Fixsen, D. J., et al. 1997, ApJ, 475, 565

Felli, M., Churchwell, E., \& Massi, M. 1984, A\&A, 136, 53

Field, D., Lemaire, J. L., Pineau des Forêts, G., et al. 1998, A\&A, 333, 280

Goeres, A., \& Sedlmayr, E., A\&A, 265, 216

de Graauw, T., Haser, L. N., Beintema, D. A., et al. 1996, A\&A, 315, L49

Hony, S., van Kerckhoven, C., Peeters, E., et al. 2000, in ISO beyond the peaks: the 2nd ISO workshop on analytical spectroscopy, ESA SP-456, to be published in A\&A

Hudgins, D. M., Sandford, S. A., \& Allamandola, L. J. 1994, J. Phys. Chem., 98, 4243

Hudgins, D. M., \& Allamandola, L. J. 1995, J. Phys. Chem., 99, 3033

Hudgins, D. M., \& Sandford, S. A. 1998, J. Phys. Chem., 102, 329

Joblin, C., Léger, A., \& Martin, P. 1992, ApJ, 393, L79

Joblin, C., d'Hendecourt, L., Léger, A., \& Défourneau, D. 1994, A\&A, 281, 923

Joblin, C., Boissel, P., Léger, A., d'Hendecourt, L., \& Défourneau, D. 1995, A\&A, 299, 835

van Kerckhoven, C., Hony, S., Peeters, E., et al. 2000, A\&A, 357, 1013

Kessler, M. F., Steinz, J. A., Anderegg, M. A., et al. 1996, A\&A, 315, L27

Klein, R., Henning, T., \& Cesarsky, D. 1999, A\&A, 343, L53

Lang, K. R. 1992, Astrophysical Data: Planets and Stars (Springer-Verlag)

Langhoff, S. R. 1996, J. Phys. Chem., 100, 2819

Laureijs, R. J., Acosta-Pulido, J., Abraham, P., et al. 1996, A\&A, 315, L313

Leach, S. 1989, in Interstellar Dust, IAU symposium no. 135, ed. L. J. Allamandola, \& A. G. G. M. Tielens (Kluwer Academic Publishers)

Lemke, D., Klass, U., Abolins, J., et al. 1996, A\&A, 315, L64

Léger, A., \& Puget, J. L. 1984, A\&A, 137, L5

Léger, A., Boissel, P., Désert, F. X., \& d'Hendecourt, L. 1989a, A\&A, 213, 351

Léger, A., d'Hendecourt, L., \& Défourneau, D. 1989b, A\&A, 216,148

Mathis, J. S., Rumpl, W., \& Nordsieck, K. H. 1977, ApJ, 217, 425

Mathis, J. S., Mezger, P. G., \& Panagia, N. 1983, A\&A, 128, 212

Mattila, K., Lemke, D., Haikala, L. K., et al. 1996, A\&A, 315, L353
Miville-Deschênes, M. A., Boulanger, F., Abergel, A., et al. 1999, in The Universe as seen by ISO, ed. P. Cox, V. Demuyt, \& M. Kessler, ESA-SP 427

Molster, F. J., van den Ancker, M. E., Tielens, A. G. G. M., et al. 1996, A\&A, 315, L373

Moutou, C., Léger, A., \& d'Hendecourt, L. 1996, A\&A, 310, 297

Moutou, C., Verstraete, L., Sellgren, K., \& Léger, A. 1999, in The Universe as seen by ISO, ed. P. Cox, V. Demuyt, \& M. Kessler, ESA-SP 427

Moutou, C., Verstraete, L., Léger, A., Sellgren, K., \& Schmidt, W. 2000, A\&A, 354, L17

Omont, A. 1986, A\&A, 164, 159

Onaka, T., Yamamura, I., Tanabe, T., Roellig, T. L., \& Yuen, L. 1996, PASJ, 48, L59

Onaka, T., Mizutani, M., Tomono, D., et al. 1999, in The Universe as seen by ISO, ed. P. Cox, V. Demuyt, \& M. Kessler, ESA-SP 427

Onaka, T. 2000, Advances in Space Research, vol. 25, issue 11, 2167

Onaka, T., Mizutani, M., Chan, K.-W., et al. 2000, in ISO beyond the peaks: the 2nd ISO workshop on analytical spectroscopy, ESA SP-456

Pauzat, F., Talbi, D., \& Ellinger, Y. 1995, A\&A, 293, 263

Pauzat, F., Talbi, D., \& Ellinger, Y. 1997, A\&A, 319, 318

Pech, C., Joblin, C., \& Boissel, P. 2000, submitted to A\&A, (PJB)

Persi, P., Cesarsky, D., Marenzi, A. R., et al. 1999, A\&A, 351, 201

Roelfsema, P. R., Cox, P., Tielens, A. G. G. M., et al. 1996, A\&A, 315, L289

Rouan, D., Léger, A., Omont, A., \& Giard, M. 1992, A\&A, 253, 498

Roche, P. F., Lucas, P. W., Hoare, M. G., Aitken, D. K., \& Smith, C. H. 1996, MNRAS, 280, 924

Rubin, R. H., Simpson, J. P., Haas, M. R., \& Erickson, E. F. 1991, ApJ, 374, 564

Salama, A. 2000, in ISO beyond point sources: Studies of extended IR emission, ed. R. J. Laureijs, K. Leech, \& M. F. Kessler, ESA SP-455

Schutte, W. A., Tielens, A. G. G. M., \& Allamandola, L. J. 1993, ApJ, 415, 397

Sellgren, K. 1984, ApJ, 277, 623

Smalley, R. E. 1983, Ann. Rev. Phys. Chem., 34, 129

Snow, T. P., \& Witt, A. N. 1996, ApJ, 468, L65

Szczepanski, J., \& Vala, M. 1993, ApJ, 414, 646

Tokunaga, A. T., Sellgren, K., Smith, R. G., et al. 1991, ApJ, 380,452

Uchida, K. I., Sellgren, K., \& Werner, M. W. 1998, ApJ, 493, L109

Uchida, K. I., Sellgren, K., Werner, M. W., \& Houdashelt, M. L. 1998, AAS, 193.6503

Uchida, K. I., Sellgren, K., Werner, M. W., \& Houdashelt, M. L. 2000, ApJ, 530, 817

Verstraete, L., \& Léger, A. 1992, A\&A, 266, 513

Verstraete, L., Puget, J. L., Falgarone, E., et al. 1996, A\&A, 315,337

van der Werf, P. P., Stutzki, J., Sternberg, A., \& Krabbe, A. 1996, A\&A, 313, 633

Whitcomb, S. E., Gatley, I., Hildebrand, R. H., et al. 1981, ApJ, 246, 416

Witteborn, F. C., Sandford, S. A., Bregman, J. D., et al. 1989, ApJ, 341, 270 